ARTICLE

\title{
Identification of distinct loci for de novo DNA methylation by DNMT3A and DNMT3B during mammalian development
}

\author{
Masaki Yagi 111,12, Mio Kabata2,3,12, Akito Tanaka², Tomoyo Ukai ${ }^{1}$, Sho Ohta ${ }^{1}$, Kazuhiko Nakabayashi ${ }^{4}$, \\ Masahito Shimizu ${ }^{3}$, Kenichiro Hata ${ }^{4}$, Alexander Meissner (10) ${ }^{5,6,7}$, Takuya Yamamoto (D) ${ }^{2,8,9,10 凶} \&$ \\ Yasuhiro Yamada (1) 1,8凶
}

De novo establishment of DNA methylation is accomplished by DNMT3A and DNMT3B. Here, we analyze de novo DNA methylation in mouse embryonic fibroblasts (2i-MEFs) derived from DNA-hypomethylated 2i/L ES cells with genetic ablation of Dnmt3a or Dnmt3b. We identify 355 and 333 uniquely unmethylated genes in Dnmt3a and Dnmt3b knockout (KO) 2i-MEFs, respectively. We find that Dnmt3a is exclusively required for de novo methylation at both TSS regions and gene bodies of Polycomb group (PcG) target developmental genes, while Dnmt3b has a dominant role on the $\mathrm{X}$ chromosome. Consistent with this, tissue-specific DNA methylation at PcG target genes is substantially reduced in Dnmt3a KO embryos. Finally, we find that human patients with DNMT3 mutations exhibit reduced DNA methylation at regions that are hypomethylated in Dnmt3 KO 2i-MEFs. In conclusion, here we report a set of unique de novo DNA methylation target sites for both DNMT3 enzymes during mammalian development that overlap with hypomethylated sites in human patients.

\footnotetext{
${ }^{1}$ Division of Stem Cell Pathology, Center for Experimental Medicine and Systems Biology, Institute of Medical Science, University of Tokyo, Tokyo 108-8639, Japan. ${ }^{2}$ Department of Life Science Frontiers, Center for iPS Cell Research and Application (CiRA), Kyoto University, Kyoto 606-8507, Japan. ${ }^{3}$ Department of Gastroenterology/Internal Medicine, Gifu University Graduate School of Medicine, Gifu 501-1194, Japan. ${ }^{4}$ Department of Maternal-Fetal Biology, National Research Institute for Child Health and Development, Tokyo 157-8535, Japan. ${ }^{5}$ Department of Genome Regulation, Max Planck Institute for Molecular Genetics, Berlin, 14195, Germany. ${ }^{6}$ Department of Stem Cell and Regenerative Biology, Harvard University, Cambridge, MA, 02138, USA. ${ }^{7}$ Broad Institute of MIT and Harvard, Cambridge, MA, 02142, USA. ${ }^{8}$ AMED-CREST, AMED 1-7-1 Otemachi, Tokyo 100-0004, Japan. ${ }^{9}$ Institute for the Advanced Study of Human Biology (WPI-ASHBi), Kyoto University, Kyoto 606-8501, Japan. ${ }^{10}$ Medical-risk Avoidance based on iPS Cells Team, RIKEN Center for Advanced Intelligence Project (AIP), Kyoto 606-8507, Japan. ${ }^{11}$ Present address: Department of Molecular Biology, Massachusetts General Hospital, Boston, MA 02114, USA. ${ }^{12}$ These authors contributed equally: Masaki Yagi, Mio Kabata. ${ }^{凶}$ email: takuya@cira.kyoto-u.ac.jp; yasu@ims.u-tokyo.ac.jp
} 
$\mathrm{D}$ NA methylation plays critical roles during mammalian development and ensures stable gene repression, genomic integrity, $\mathrm{X}$ chromosome inactivation (XCI), and preservation of genomic imprinting ${ }^{1-3}$. Three conserved DNA methyltransferases, DNMT3A, DNMT3B, and DNMT1, are responsible for de novo establishment and maintenance of DNA methylation patterns ${ }^{4}$. The significance of DNA methyltransferases for mammalian development is highlighted by the fact that genetic deletions of any of these enzymes have lethal phenotypes in mice ${ }^{5,6}$. Moreover, dysregulation of DNMTs is linked to several human diseases, including acute myeloid leukemia (AML) and immunodeficiency, centromere instability, and facial anomalies (ICF) syndrome, indicating that DNA methyltransferases are essential for normal mammalian development and proper maintenance of somatic cells ${ }^{7,8}$.

Previous studies revealed the epigenetic mechanisms that establish the strict methylome integrity required for normal development. After epigenetic reprogramming in preimplantation embryos, the DNA methyltransferases DNMT3A and DNMT3B, in conjunction with their nonenzymatic cofactor DNMT3L, mediate global de novo DNA methylation during embryonic and extraembryonic development ${ }^{5,9,10}$. DNMT3A and DNMT3L establish genomic imprinting in gametes ${ }^{11-13}$, whereas DNMT3B and DNMT3L are required for unique DNA methylation patterns in placental progenitors ${ }^{10}$. The genomic enrichment patterns of DNMT3A differ from those of DNMT3B in mouse embryonic stem (ES) cells, suggesting that each de novo methyltransferase has unique targets during development ${ }^{14,15}$. Indeed, individual genetic ablation of these methyltransferases results in lethal phenotypes at different developmental stages 5 . However, it remains unclear whether the differential targeting of DNMT3 enzymes is responsible for locus-specific de novo DNA methylation during embryonic development.

To comprehensively identify the target sites for de novo DNA methylation by the DNMT3 enzymes, in the present study, we took advantage of female mouse ES cells established in the presence of MEK and Gsk3 inhibitors, which lack most DNA methylation $(2 \mathrm{i} / \mathrm{L} \text { ES cells })^{16}$. In combination with genetic ablation of Dnmt3a or Dnmt3b, here we report unique de novo DNA methylation target sites for both DNMT3 enzymes during embryonic development.

\section{Results}

DNA hypomethylated ESCs for de novo methylation analysis. Given the early embryonic lethal phenotype of Dnmt $3 b$ knockout (KO) mice, ES cells could be a powerful tool for tracking and comparing the de novo methylation activity of DNMT3s during embryonic development (Fig. 1a). It should be noted, however, that mouse ES cells cultured in serum and leukemia inhibitory factor (LIF) (S/L ES cells) exhibit global DNA hypermethylation relative to inner cell mass (ICM) cells, which are the in vivo counterpart of ES cells ${ }^{17}$. Indeed, de novo methylated regions at post-implantation epiblasts in vivo are already methylated in S/L ES cells (Fig. 1b, c). Moreover, Polycomb group (PcG) target transcription factor genes, which often include key developmental genes, are highly methylated in S/L ES cells, but still hypomethylated in both ICM and epiblast (Fig. 1d and Supplementary Fig. 1a), precluding the use of S/L ES cells for analysis of de novo methylation at early developmental stages. In a previous study, we showed that mouse ES cells established under 2i/L (MEK inhibitor, Gsk3 inhibitor, and LIF) culture conditions (2i/L ES cells) exhibit a substantial reduction in global DNA methylation levels ${ }^{16}$. Female 2i/L ES cells lack DNA methylation at most sites, including the PcG target genes (Fig. 1b-d and Supplementary Fig. 1a). Collectively, these findings indicated that hypomethylated female
2i/L ES cells represent a powerful tool for de novo methylation during early embryonic development.

In this study, to identify the unique targets of de novo methylation by the DNMT3 enzymes, we used female mouse 2i/L ES cells with targeted deletions in either Dnmt3a or Dnmt3b (Fig. 1a). Dnmt3 KO 2i/L ES cells grow well and are morphologically normal under $2 \mathrm{i} / \mathrm{L}$ culture conditions ${ }^{16}$. In addition, they efficiently contribute to chimeric embryos as efficiently as wild-type (WT) 2i/L ES cells, indicating that Dnmt3 KO 2i/L ES cells retain pluripotency and the ability to differentiate into somatic cells in vivo ${ }^{16}$. To compare the targets of each DNA methyltransferase, we established mouse embryonic fibroblasts (MEFs) from chimeric mice derived from female Dnmt3 KO 2i/L ES cells (Dnmt3 KO 2i-MEFs), followed by antibiotic selection, and assessed DNA methylation profiles by whole-genome bisulfite sequencing (WGBS) and target-captured MethylC-seq analysis (Supplementary Fig. 1b). Considering that the $5 \mathrm{hmC}$ level is negligible in ESCs ${ }^{18}$, we regarded both $5 \mathrm{hmC}$ and $5 \mathrm{mC}$ as $5 \mathrm{mC}$ in this study.

CpG methylation on all chromosomes was lower in both Dnmt3a and Dnmt3b KO 2i-MEFs than in WT 2i-MEFs (Fig. 1e and Supplementary Fig. 1c). Extending the analysis to various genomic features, including transposable elements, we found that both Dnmt3a and Dnmt3b KO decreased DNA methylation levels on all elements (Fig. 1f, g). Consistent with a previous study ${ }^{19}$, Dnmt3b KO 2i-MEFs exhibited a clear reduction in DNA methylation on transposable elements relative to WT or Dnmt3a KO 2i-MEFs (Fig. 1g). As shown in our previous study ${ }^{16}, 2 \mathrm{i}-$ MEFs exhibited global loss of genomic imprinting regardless of Dnmt3 status (Supplementary Fig. 1d). When we divided all CpG sites into three categories according to DNA methylation levels [hypermethylated $(>0.8)$, intermediate $(0.2-0.8)$, and hypomethylated $(<0.2)$ sites], we found that hypermethylated $\mathrm{CpG}$ sites were less abundant in Dnmt3b KO 2i-MEFs, whereas Dnmt3a KO 2iMEFs exhibited relatively modest reduction (Fig. 1h). The reduced methylation level in Dnmt3b KO 2i-MEFs was observed at regions marked by various histone marks, such as H3K36me3 and H3K9me3 (Supplementary Fig. 2a) ${ }^{14}$. We also found that Dnmt3b KO epiblasts exhibit reduced CpG methylation levels at de novo methylated regions during peri-implantation (from ICM to post-implantation epiblasts) relative to WT or Dnmt3a KO epiblasts (Supplementary Fig. 2b). Together, these results suggest that DNMT3B de novo DNA methylation activity is predominant in most genomic regions during early development.

Consistent with a previous report ${ }^{20}$, methylation levels at CGIs on the X chromosome were markedly lower in Dnmt3b KO 2iMEFs than in Dnmt3a KO 2i-MEFs (Fig. 1i and Supplementary Fig. 2c) (3a KO vs WT: $p=3.6 \times 10^{-1}, 3 b$ KO vs WT: $p=1.2 \times$ $10^{-15}$; two-sided, Kolmogorov-Smirnov test), suggesting that Dnmt3b is largely responsible for de novo CGI methylation on the $\mathrm{X}$ chromosome. Non-CGI sites were also less methylated in Dnmt3b KO 2i-MEFs, which was more pronounced on the $\mathrm{X}$ chromosome than on autosomes (Fig. 1i and Supplementary Fig. 2c-e) (3a KO vs WT: $p<2.2 \times 10^{-16}, 3 \mathrm{~b}$ KO vs WT: $p<2.2 \times$ 10 16 ; two-sided, Kolmogorov-Smirnov test). In sharp contrast, Dnmt3a KO 2i-MEFs did not exhibit an apparent reduction in non-CGI methylation on the $\mathrm{X}$ chromosome (Fig. 1i and Supplementary Fig. 2c). Collectively, these results suggest that DNMT3B is the enzyme predominantly responsible for global de novo methylation on the $\mathrm{X}$ chromosome during $\mathrm{XCI}$.

Target genes of de novo methylation by DNMT3A and DNMT3B. We next sought to identify, in an unbiased manner, unique target loci of DNMT3A- and DNMT3B-mediated de novo DNA methylation during embryonic development. To this end, 
a

DNA hypomethylated ESCs

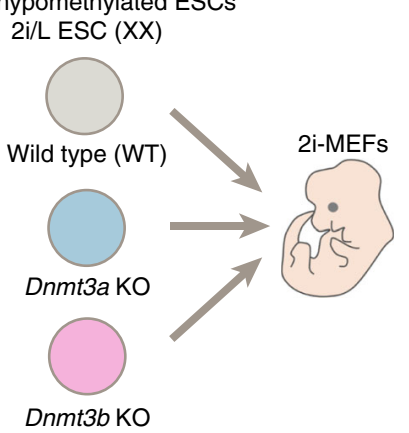

C

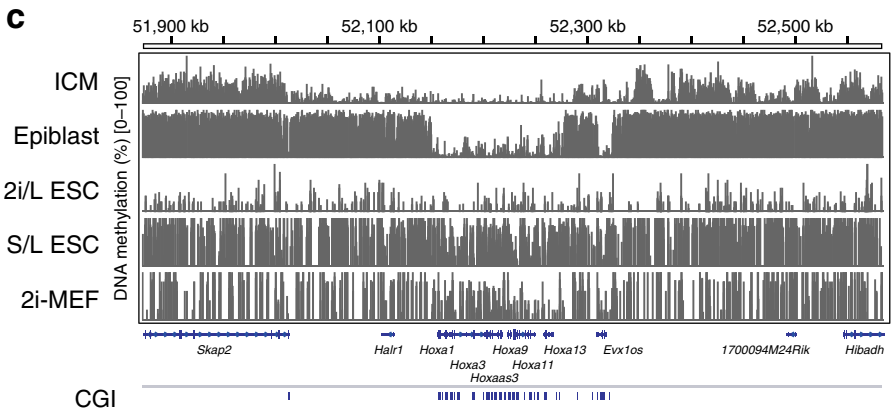

e

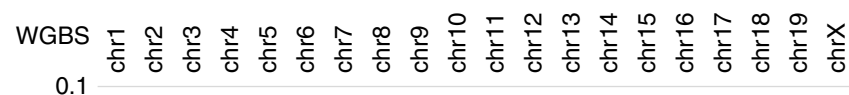

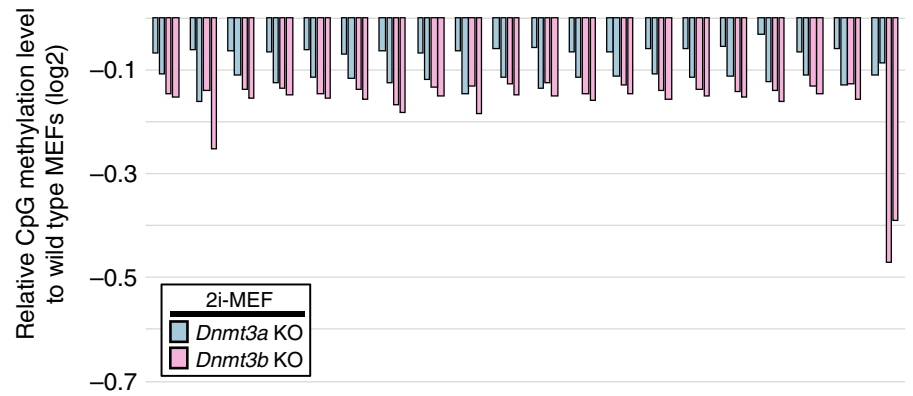

g

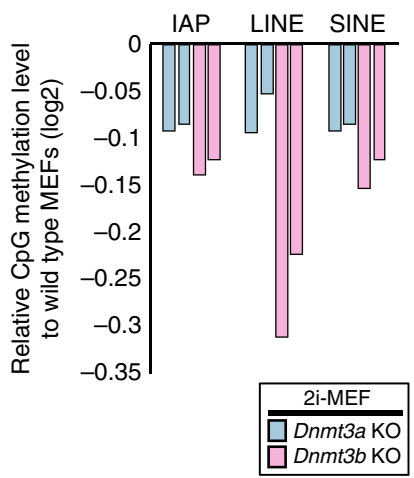

DNA methylation Methyl-seq, WGBS

RNA-seq

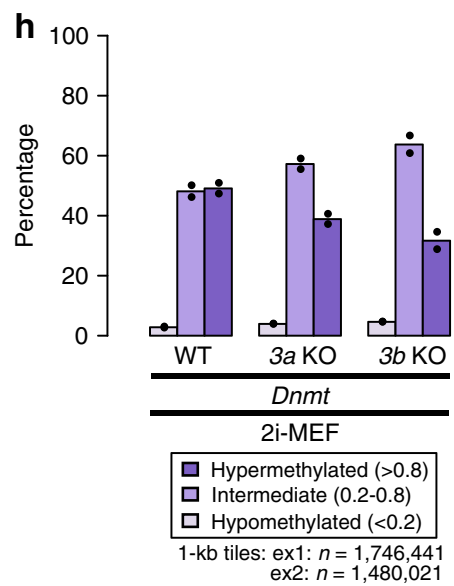

b

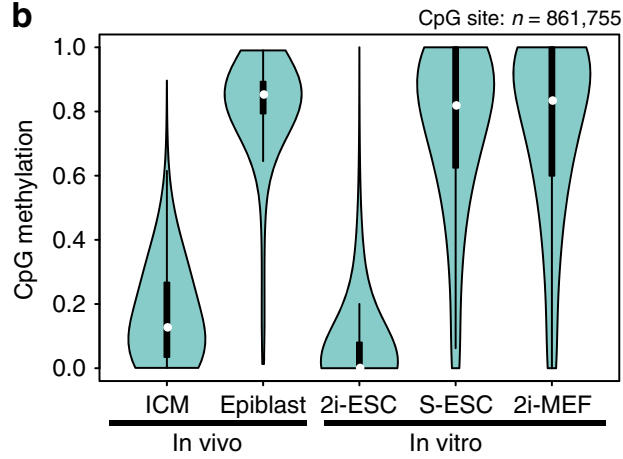

d

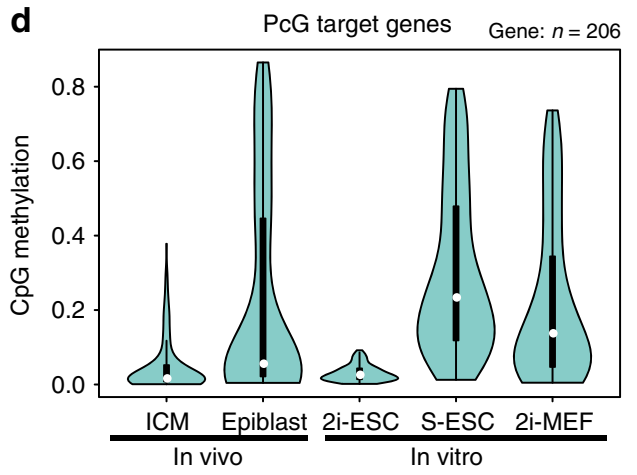

f

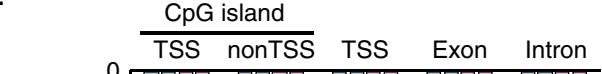

we first identified differentially methylated tiles (DMT; nonoverlapping $1 \mathrm{k}$ tiles) that differed significantly between WT $2 \mathrm{i}$ MEFs and each KO 2i-MEFs by using WGBS data. Consistent with previous reports ${ }^{5,21}$, DNA methylation levels at most regions did not significantly differ between Dnmt3a and Dnmt3b KO 2iMEFs, but we identified thousands of DMTs in either of the KO i
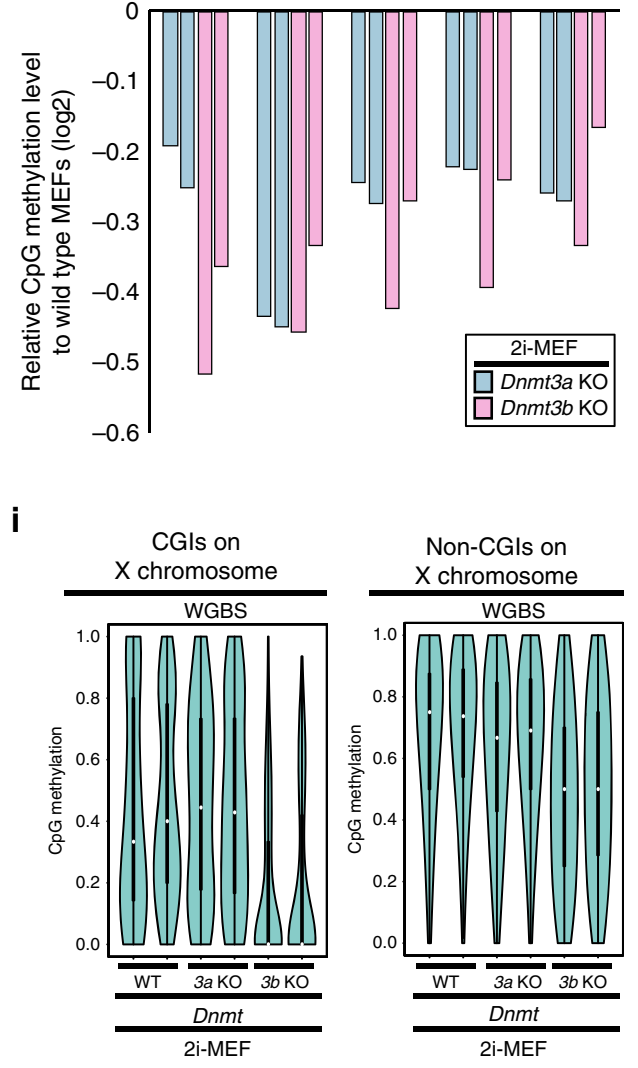

(4308 and 7357 DMTs in Dnmt3a and Dnmt3b KO 2i-MEFs, respectively) (Fig. 2a and Supplementary Data 1). A small subset of DMTs $(n=143)$ exhibited a modest reduction in DNA methylation in both Dnmt3a KO and Dnmt3b KO 2i-MEFs, suggesting that these loci are sufficiently methylated only when DNMT3A and DNMT3B are both present (Fig. 2a and 
Fig. 1 DNA hypomethylated ES cells for de novo methylation analysis. a Schematic of experimental design. Either Dnmt3a or Dnmt3b was disrupted by CRISPR/Cas9 in female 2i/L ES cells ${ }^{16}$. b CpG methylation levels at loci that were differentially methylated between ICM and epiblast (see the methods section for details) in 2i/L ES cells and S/L ES cells. WGBS data were used for the analysis. White dots indicate median methylation levels. Black bars and the lines stretched from the bar represent IQR and the lower/upper adjacent values $( \pm 1.5 \mathrm{IQR})$, respectively. ICM and epiblast data were obtained from GSE84236 ${ }^{10}$. Data of 2i/L ES cells, S/L ES cells, and 2i-MEFs are from GSE84165 ${ }^{16}$. c $\mathrm{CpG}$ methylation levels at a representative genomic region including the Hoxa cluster, as determined by WGBS. Each bar indicates a CpG site, and the height of the bar represents methylation percentage (0-100\%). Locations of genes and CpG islands (CGIs) are indicated below. Data are from GSE8423610 and GSE84165 ${ }^{16}$. d CpG methylation levels at PcG target developmental genes in ICM, epiblast, 2i/L ES cells, S/L ES cells, and 2i-MEFs, as determined by WGBS. Data were obtained from GSE84236 ${ }^{10}$ and GSE84165 $5^{16}$. e Relative CpG methylation level [ $\log _{2}$ (fold change)] at each chromosome in Dnmt3 KO 2i-MEFs vs. wild-type 2i-MEFs, as determined by WGBS. Data from two independent experiments are shown. f Relative CpG methylation levels $\left[\log _{2}\right.$ (fold change)] at CGls, promoters [Transcription Start Site (TSS) \pm 1000 bp],

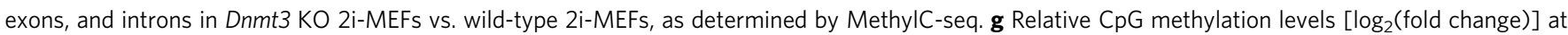
transposable elements (IAPs, LINEs, and SINEs) in Dnmt3 KO 2i-MEFs vs. wild-type 2i-MEFs by WGBS. h: Fraction of hypermethylated (>0.8), intermediate (0.2-0.8), and hypomethylated $(<0.2) \mathrm{CpG}$ sites in wild-type and each type of KO 2i-MEFs. WGBS data (CpG sites at $\geq 5 \times$ coverage) were used for this analysis. i CpG methylation levels at CGIs and non-CGIs on the X chromosome in WT, Dnmt3a KO, and Dnmt3b KO 2i-MEFs, as determined by WGBS. White dots indicate median methylation levels.

Supplementary Data 1). To uncover the specific target genes of each methyltransferase, we used MethylC-seq data that are enriched and have high coverage reads in gene regions. Notably, we identified 355 and 333 specific target genes of DNMT3A and DNMT3B, respectively (Fig. 2b, c and Supplementary Fig. 3a, b) (Supplementary Data 2-5). Gene ontology (GO) analyses revealed that DNMT3B-specific genes are involved in synaptonemal complex assembly (e.g., Tex12, Syde1, and Meioc), plasma membrane (e.g., Pcdha/b family), and cell proliferation in forebrain (e.g., Wnt7a, Wnt7b, Wnt3a, and Zeb2) (Supplementary Fig. 3c, and Supplementary Data 4 and 5). Our analysis identified the previously reported DNMT3B-specific targets, $P c d h a / b$ family genes $^{22}$, confirming that our strategy can successfully identify the target genes of the de novo methyltransferase (Supplementary Fig. 3a). By contrast, DNMT3A target genes included many transcription factors that are essential for normal development, including genes of the Hox, Gata, and Cbx families (Fig. 2c and Supplementary Data 3 and 4). Consistent with this, GO analyses indicated that DNMT3A target genes are involved in sequencespecific DNA binding (e.g., Nr6a1, Gata2, Hoxc9, and Pou5f1), transcription (e.g., $Z f p 46$, Barxl, and Osr1), and pharyngeal system development (e.g., Gata3, Six1, and Tbx1) (Supplementary Fig. 3d). Because the DNMT3A target genes included many developmental genes that are also known as PcG target transcription factor genes ${ }^{23}$, we next hypothesized that DNMT3A regulates de novo DNA methylation at PcG target developmental genes during embryonic development. Notably, most of the PcG target genes that acquire de novo DNA methylation during embryonic development were strikingly hypomethylated in Dnmt3a KO 2i-MEFs (Fig. 2d and Supplementary Fig. 3e) [3a KO vs WT (mean methylation levels): $p=5.6 \times 10^{-48}, 3 \mathrm{~b}$ KO vs WT (mean methylation level): $\mathrm{p}=8.2 \times 10^{-16}$, Wilcoxon signed-rank test]. For example, our data showed that all Hox gene clusters (Hoxa, Hoxb, Hoxc, and Hoxd) located on different chromosomes, as well as other PcG target transcription factors, were specifically methylated by DNMT3A (Fig. 2c, Supplementary Fig. 3b, and Supplementary Data 3). The reduced DNA methylation in Dnmt3a KO 2i-MEFs was observed in both short and long genes (Supplementary Fig. 4a). To confirm DNMT3Amediated de novo methylation, we next examined the effect of ectopic Dnmt3a expression on reduced methylation at PcG target developmental genes in Dnmt3a KO cells. In these experiments, we introduced a doxycycline (Dox)-inducible Dnmt3al expression vector into Dnmt3a KO 2i/L ES cells, and then differentiated them into epiblast-like cells (EpiLCs) (Supplementary Fig. 4b). Forced expression of Dnmt3a1 in Dnmt3a KO cells increased de novo DNA methylation at Hoxa1 and Meis1 to a level similar to that observed in WT cells (Supplementary Fig. 4c, d and Source
Data file). Based on these findings, we concluded that Dnmt3a confers de novo DNA methylation at PcG target developmental genes upon ES cell differentiation.

To more thoroughly examine DNMT3A-mediated de novo methylation at PcG target developmental genes, we divided the region of each PcG target gene into the Transcription Start Site (TSS) region (TSS $\pm 1000 \mathrm{bp}$ ) and gene body [TSS to Transcription End Site (TES)] and analyzed the methylation patterns (Fig. 2e). The TSS regions of a subset of PcG target developmental genes exhibited lower levels of de novo methylation in Dnmt3a KO 2i-MEFs than in WT 2i-MEFs (Fig. 2e) [3a KO vs WT (mean methylation levels, TSS): $p<2.2 \times 10^{-16}, 3 \mathrm{~b}$ KO vs WT (mean methylation levels, TSS): $p=5.4 \times 10^{-14}$; two-sided, Wilcoxon signed-rank test]. In contrast to the repressive role of DNA methylation at TSS regions, gene body methylation is generally associated with active transcription ${ }^{24}$. Consistent with this, gene bodies of more highly expressed PcG target developmental genes tended to have higher methylation levels in 2i-MEFs (Fig. 2e). In addition, DNMT3B binds to gene bodies of actively transcribed genes marked by $\mathrm{H} 3 \mathrm{~K} 36 \mathrm{me} 3$, and is responsible for de novo DNA methylation at the gene bodies ${ }^{14,25}$. Indeed, H3K36me3-marked regions were less methylated in $D n m t 3 b$ KO 2i-MEFs than in Dnmt3a KO 2i-MEFs (Supplementary Fig. 2a). Importantly, however, gene bodies of PcG target genes were less methylated in Dnmt3a KO 2i-MEFs than in Dnmt3b KO 2i-MEFs (Fig. 2e and Supplementary Fig. 5a) [ 3 a KO vs WT (Gene body): $p<2.2 \times$ $10^{-16}$, 3b KO vs WT (Gene body): $p=8.9 \times 10^{-3}$; two-sided, Wilcoxon signed-rank test]. The reduced methylation at gene bodies in Dnmt3a KO 2i-MEFs was similarly observed at those distant from TSS (Supplementary Fig. 5b), indicating that the observed changes are not always associated with close proximity of $\mathrm{CpG}$ islands linked to promoters. Collectively, these results suggest that, in sharp contrast to other genes, gene bodies of PcG target developmental genes are preferentially methylated by DNMT3A, consistent with a recent study demonstrating that DNMT3A1, a longer isoform of DNMT3A, preferentially localizes to the methylated shores of bivalent CGIs in ES cells ${ }^{15}$. Given that DNMT3A, together with DNMT3L, establishes genomic imprinting in gametes, we examined the involvement of DNMT3L in DNMT3A-mediated de novo methylation at PcG target genes. However, methylation levels of PcG target genes were comparable between Dnmt3l KO and WT E14.5 MEFs (Supplementary Fig. 5c), demonstrating that DNMT3L is dispensable for DNMT3A-mediated methylation at PcG target genes during embryonic development.

Differential binding patterns of DNMT3A1 and DNMT3B1. To obtain mechanistic insights into the locus specificity of 
a

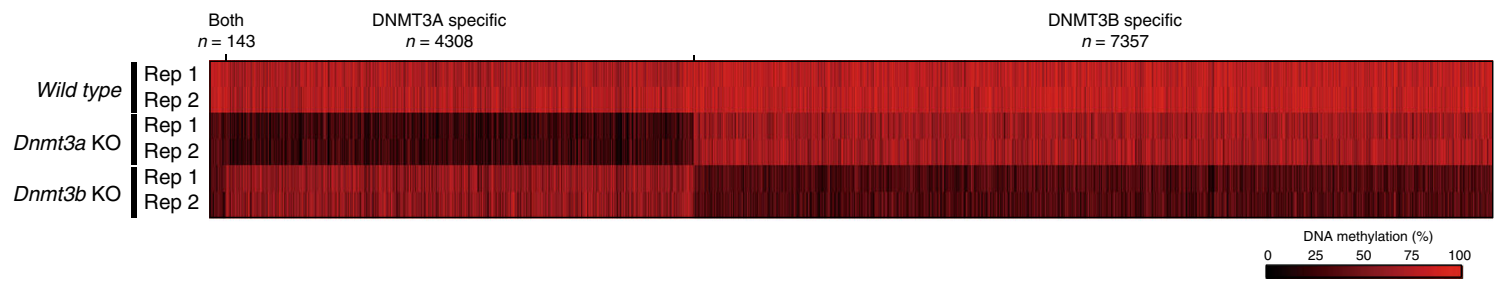

b
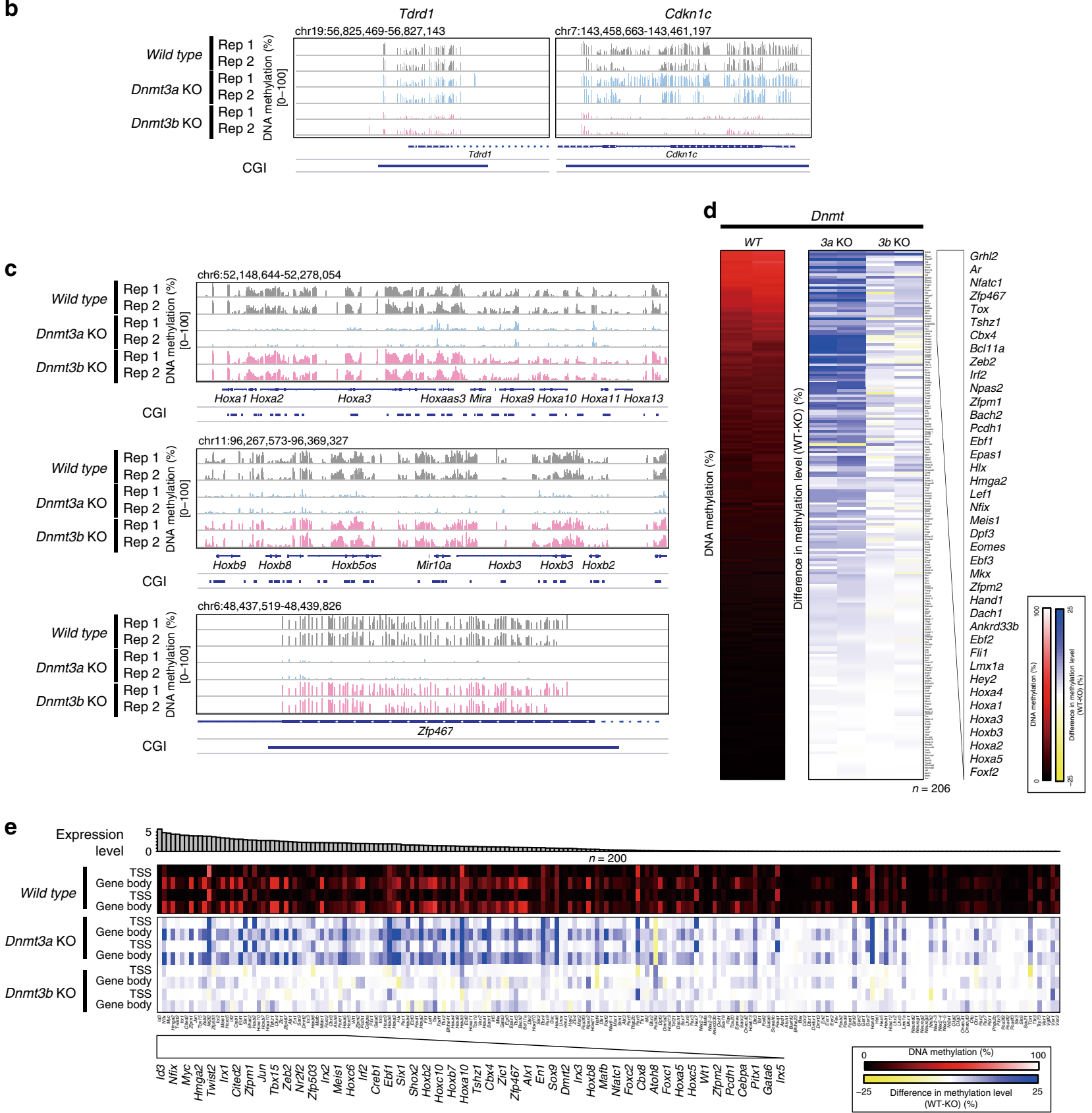

DNMT3 enzymes for de novo methylation, we examined genomic enrichment patterns of DNMT3A and DNMT3B by analyzing ChIP-seq datasets ${ }^{14,15}$. Consistent with previous reports demonstrating that DNMT3B is responsible for gene body methylation of actively transcribed genes ${ }^{14,25}$, we confirmed enrichment of DNMT3B1, but not DNMT3A1, in gene bodies (Fig. 3a). Notably, DNMT3A, and especially DNMT3A1, was enriched at DNMT3A target genes (Fig. 3a and Supplementary Fig. 6a). By contrast, enrichment of DNMT3B1 was not altered at target genes of DNMT3B-mediated de novo methylation (Fig. 3a), suggesting the existence of distinct mechanisms governing the locus-specific activity of de novo methyltransferases. Considering that DNMT3A mediates de novo methylation at PcG target developmental genes, we next analyzed the binding patterns of the DNMT3 enzymes on the PcG target genes. We observed prominent enrichment of DNMT3A1 binding on PcG target developmental genes, including enrichment in gene bodies (Fig. 3b). Remarkably, DNMT3B1 binding was reduced on the 
Fig. 2 Target genes of de novo methylation by DNMT3A and DNMT3B. a Heatmap of CpG methylation levels within $1 \mathrm{~K}$ tiled regions in which CpG methylation levels differed between the WT and each Dnmt3 KO. Differentially methylated $1 \mathrm{~K}$ tiled regions are identified using the Bioconductor DSS package with methylation status of nonoverlapping $1 \mathrm{~K}$ tiles that have $\geq 10$ read coverages at $\mathrm{CpG}$ sites within a tile across all samples (see the methods section in detail). b Representative loci of DNMT3B-specific target sites in 2i-MEFs. Each bar indicates a CpG site, and the height of the bar represents methylation percentage, as determined by MethyIC-seq (0-100\%). Locations of genes and CGIs are indicated below. c Representative loci of DNMT3Aspecific target sites in 2i-MEFs. Essential developmental genes are frequently unmethylated in Dnmt3a KO 2i-MEFs. Each bar indicates a CpG site, and bar height represents methylation percentage by MethylC-seq (0-100\%). Locations of genes and CGIs are indicated below. $\mathbf{d}$ The left panel shows mean DNA methylation levels at CpG sites within each PcG target developmental gene ${ }^{23}$ [from TSS to Transcription End Site (TES)] in WT 2i-MEFs from two independent experiments. The right panel shows methylation difference between WT and each Dnmt3 KO 2i-MEFs from two independent experiments. Genes are sorted by the mean methylation levels in the WT. Color scales indicate DNA methylation levels and their differences, respectively. e The upper panel shows mean DNA methylation levels at CpG sites within TSS \pm 1000 bp and gene body (TSS-TES) of each PcG target gene in WT 2i-MEFs from two independent experiments. The lower panel shows methylation difference between WT and each type of Dnmt3 KO 2i-MEFs from two independent experiments. Color scale indicates DNA methylation levels and their difference, respectively. PcG genes are ordered based on expression levels (FPKM values) in WT 2i-MEFs (top panel).

PcG target genes ${ }^{23}$ (Fig. 3b), indicating that DNMT3B1 is precluded from these loci. Among DNMT3A target genes, further enrichment of DNMT3A1 was observed on PcG target developmental genes (Fig. 3c), and reduced binding of DNMT3B1 was detected exclusively at DNMT3A target genes that were also PcG target genes (Fig. 3c), highlighting unique binding patterns of DNMT3 enzymes at PcG target loci. Together, these results suggest that the preferred physical interactions of DNMT3A and DNMT3B are associated with the locus-specific activity of the enzymes at PcG target developmental genes.

Derepression of PcG target genes in Dnmt3a KO 2i-MEFs. Given that DNA methylation at CGIs linked to TSS regions are associated with gene repression, we next investigated the functional implications of DNMT3-mediated de novo methylation on gene expression during embryonic development. Because Dnmt3b KO 2i-MEFs exhibited global CGI hypomethylation at the $\mathrm{X}$ chromosome, we first performed RNA-seq analysis to compare global gene expression profiles of X-linked genes between WT and both types of Dnmt KO 2i-MEFs. Despite the significant reduction in CGI methylation in Dnmt3b KO 2i-MEFs, expression levels of $\mathrm{X}$-linked genes were comparable among WT, Dnmt3a KO, and Dnmt3b KO ( $3 a$ KO: $p=7.6 \times 10^{-1}, 3 b$ KO: $\left.p=8.2 \times 10^{-1}\right)$ (Fig. 4a), suggesting that DNA methylation-independent mechanisms ensure dosage compensation of X-linked genes in Dnmt3b KO 2i-MEFs ${ }^{26}$. We also examined expression of PcG target developmental genes in WT and Dnmt KO 2i-MEFs. Although reduced CGI methylation at PcG target developmental genes was exclusively detectable in Dnmt3a KO 2i-MEFs, expression levels of most PcG target developmental genes were not altered in Dnmt3a KO 2i-MEFs ( $3 a \mathrm{KO}: p=7.3 \times 10^{-1}, 3 b \mathrm{KO}: p$ $=6.5 \times 10^{-1}$ ) (Fig. 4b). However, when we focused on the TSS regions of PcG target developmental genes expressed at low levels, which were methylated in WT $2 \mathrm{i}-\mathrm{MEF}$ ( $>15 \%)$, we found that a small subset of the PcG target genes (e.g., Pax8) was modestly derepressed in Dnmt3a KO 2i-MEFs, accompanied by loss of DNA methylation at TSS regions (Fig. 4c-e and Supplementary Fig. 6b). To further investigate DNMT3A-mediated repression of PcG target developmental genes, we also obtained MEFs from Dnmt3a WT and null embryos by crossing Dnmt3a heterozygous KO mice. Importantly, derepression of Pax 8 was confirmed in Dnmt3a KO MEFs (Supplementary Fig. 6c). Collectively, these data indicate that DNMT3A-mediated DNA methylation plays a role in stable silencing of a subset of PcG target developmental genes.

Tissue-specific methylation at PcG target genes by DNMT3A. Our previous study demonstrated that female $2 \mathrm{i} / \mathrm{L}$ ES cells exhibit a massive erasure of genomic imprints and harbor an impaired autonomous developmental potential ${ }^{16}$, which raised the possibility that the observed changes in DNA methylation are not directly associated with DNMT3 de novo DNA methylation activity. To investigate DNMT3A-mediated de novo methylation during embryonic development in vivo, we obtained various tissues (brain, limb, heart, lung, and liver) from Dnmt3a WT and null embryos at E14.5 (Fig. 5a, b). We then performed MethylCseq analysis to compare DNA methylation patterns at PcG target developmental genes in different tissues (Supplementary Fig. 1b). Notably in this regard, PcG target genes in different tissues exhibited distinct DNA methylation levels in WT embryos. For example, the Hoxb cluster is methylated in the heart and liver, but remains unmethylated in the brain (Fig. 5c). Notably, genetic ablation of Dnmt $3 a$ resulted in a reduction in DNA methylation at the Hoxb cluster in the heart and liver (Fig. 5c). Moreover, other PcG target developmental genes such as Chromo, T-box, and Hox families also exhibited tissue-specific methylation patterns in WT organs, but were hypomethylated in Dnmt3a KO tissues (Fig. 5d-e and Supplementary Fig. 7). Conventional bisulfite sequencing analysis confirmed that the tissue-specific methylation at Meis1 is mediated by DNMT3A (Supplementary Fig. 8a). Consistent with the notion that PcG target developmental genes are regulated by cooperative epigenetic mechanisms, most PcG target genes were expressed at similar levels in WT and Dnmt3a KO tissues (Fig. 5f and Supplementary Fig. 8b). Notably, gene bodies of transcriptionally active PcG target developmental genes were highly methylated in both the heart and liver, but were less methylated in Dnmt3a KO tissues (Supplementary Fig. 8c), affirming that de novo methylation at gene bodies of PcG target developmental genes is mediated by DNMT3A in vivo. Taken together, these results underscore the essential role of DNMT3A in establishing tissue-specific DNA methylation patterns at PcG target developmental genes during embryonic development.

Reduced DNA methylation in patients with DNMT3 mutations. Finally, we investigated the unique functions of DNMT3A and DNMT3B in humans. Somatic inactivating mutations of DNMT3A and DNMT3B have been reported in AML ${ }^{27}$ and ICF syndrome patients ${ }^{7}$, respectively. To investigate the possible link between an impaired DNMT3 function and altered DNA methylation in human diseases, we first analyzed DNA methylation status at mouse DNMT3B target genes in ICF patients harboring a $D N M T 3 B$ mutation. Human homologs of a subset of mouse DNMT3B target genes were similarly hypomethylated in DNMT3B mutant ICF patients relative to control patients (Fig. 6a). Importantly, in addition to the $P C D H$ family of genes, previously reported to be hypomethylated in 

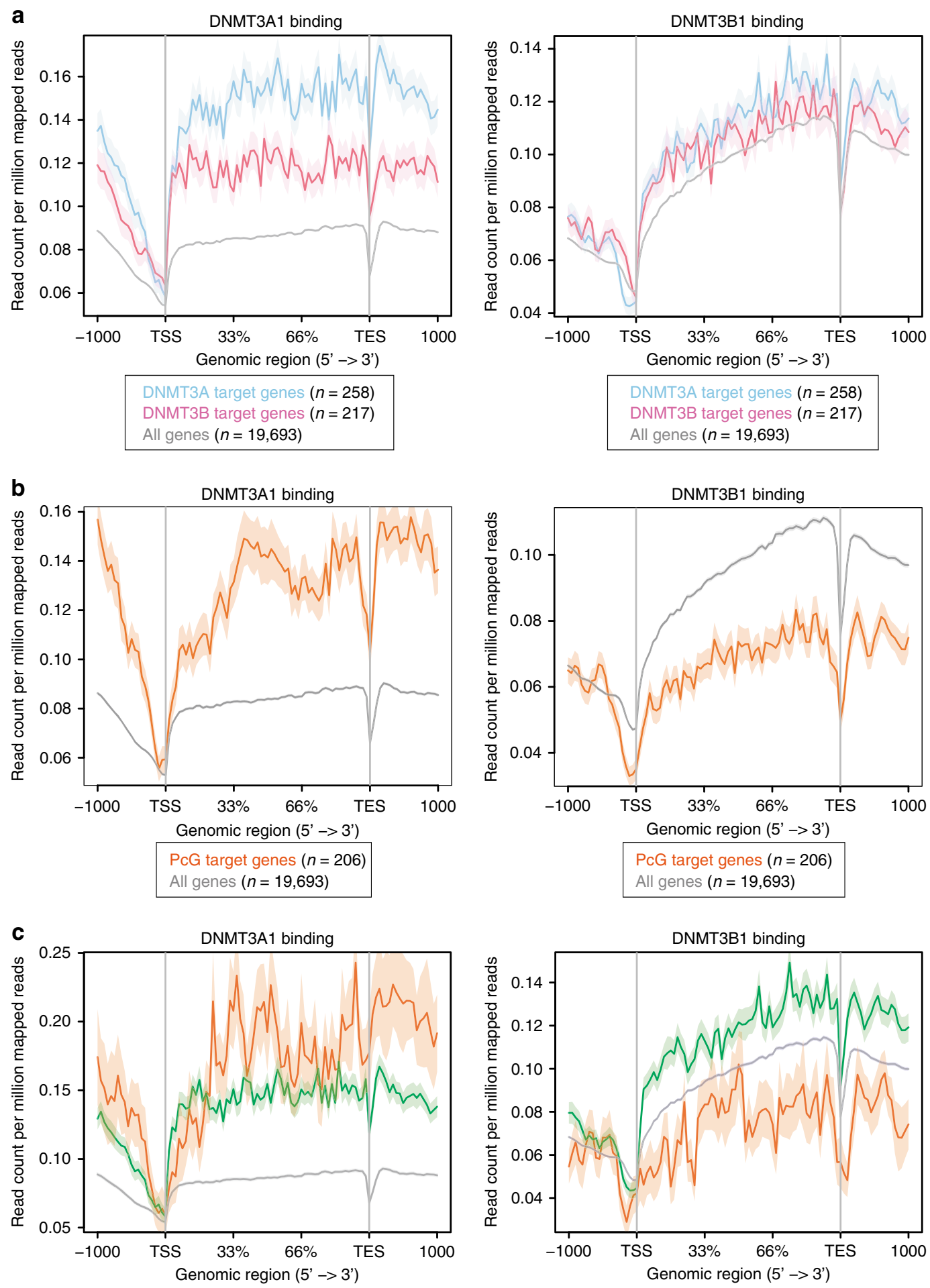

DNMT3A target PcG genes $(n=32)$
DNMT3A target non-PcG genes $(n=226)$
All genes $(n=19,693)$

$$
\begin{aligned}
& \text { DNMT3A target PcG genes }(n=32) \\
& \text { DNMT3A target non-PcG genes }(n=226) \\
& \text { All genes }(n=19,693)
\end{aligned}
$$

Fig. 3 Differential binding patterns of DNMT3A1 and DNMT3B1. a DNMT3A1 and DNMT3B1 binding to target genes for de novo methylation. ChIP-seq data were obtained from GSE57413 and GSE9652914,15. Shading represents the standard error of the mean (SEM). b DNMT3A1 and DNMT3B1 binding to PcG target developmental genes. Note that DNMT3A1 preferentially binds to PcG target genes, whereas DNMT3B1 is excluded from these genes. Shading represents SEM. c DNMT3A1 and DNMT3B1 binding to DNMT3A target genes that are also PcG target genes. Further enrichment of DNMT3A1 was observed on PcG target genes. Note that reduced binding of DNMT3B1 was exclusively detected at PcG target genes among DNMT3A target genes. Shading represents SEM. 
a

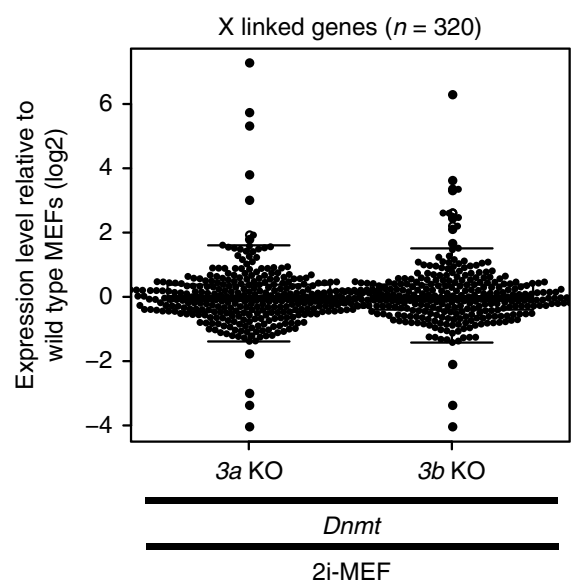

c

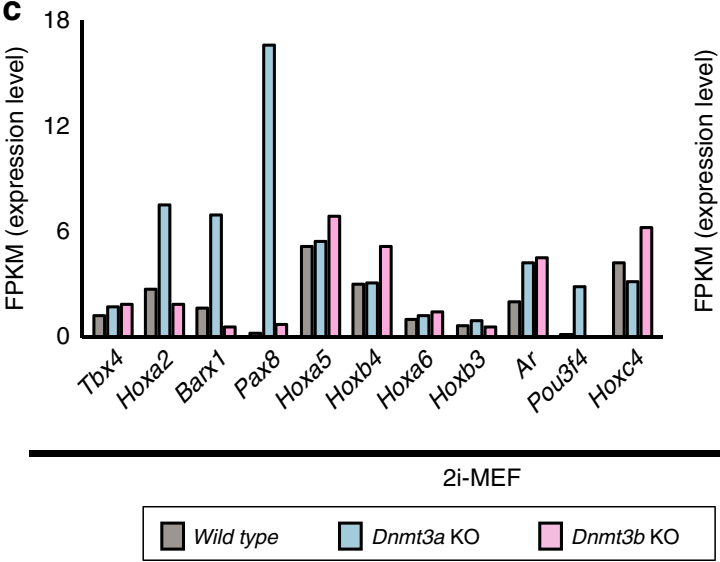

b
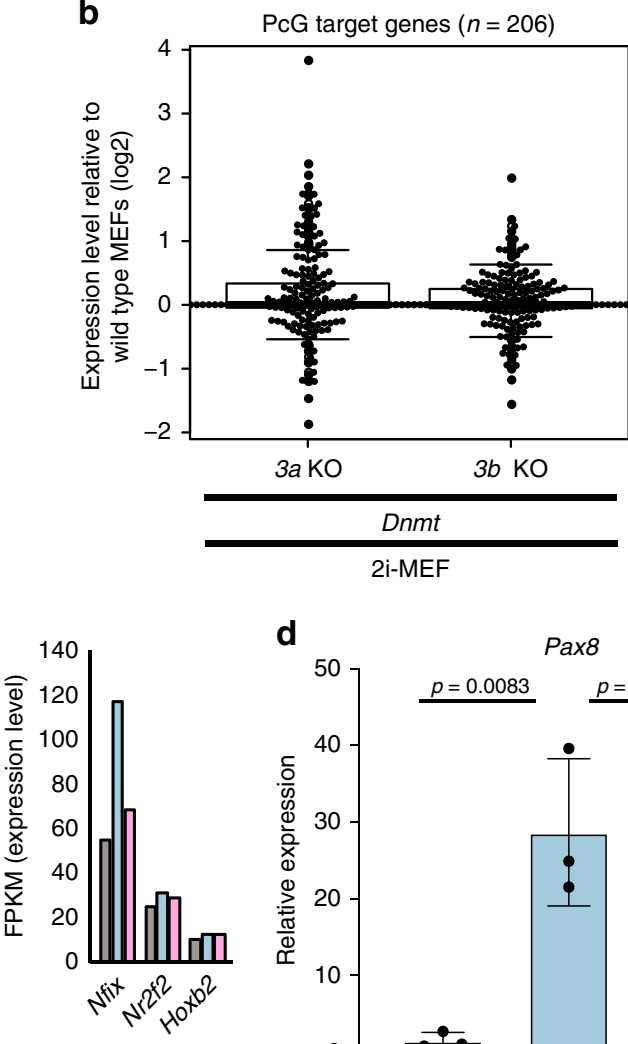

d

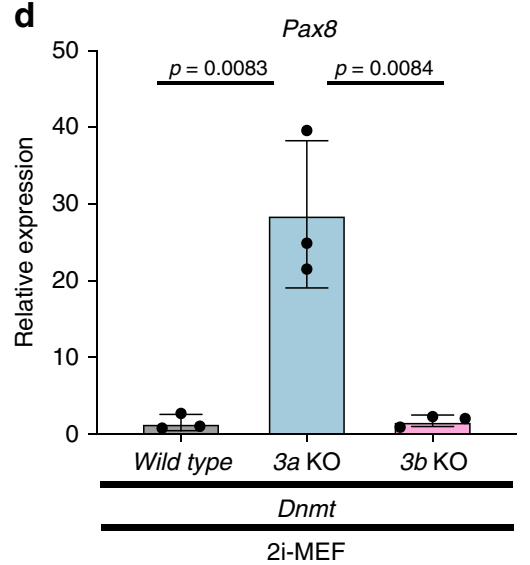

Pax8

(1) 24,47

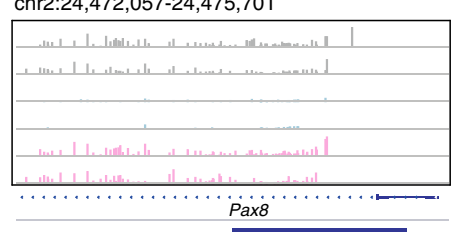

Fig. 4 Derepression of PcG target genes in Dnmt3a KO 2i-MEFs. a Relative gene expression levels (FPKM +1$)$ of $X$-linked genes $(n=320)$ in Dnmt3 KO 2i-MEFs $(n=1)$ vs. WT 2i-MEFs $(n=1)$, as determined by RNA-seq. Note that expression levels of most X-linked genes were comparable, regardless of Dnmt3 status. Solid lines in each box indicate the median. The bottom and top of the boxes are lower and upper quartiles, respectively. Whiskers extend to \pm 1.5 IQR. b Relative gene expression levels (FPKM +1$)$ of PcG target developmental genes $(n=206)$ in Dnmt3 KO 2i-MEFs $(n=1)$ vs. WT 2i-MEFs $(n=1)$, as determined by RNA-seq. Note that most PcG target genes were expressed at similar levels. Only a small subset of genes was modestly upregulated in Dnmt3 KO 2i-MEFs. c Expression level (FPKM) of PcG target genes, as determined by RNA-seq. Genes methylated more than $15 \%$ at TSS \pm 1000 bp in WT 2i-MEFs are shown. A small subset of genes was modestly derepressed in Dnmt3a KO 2i-MEFs relative to the other cell types. $\mathbf{d}$ qRT-PCR analysis for Pax8 in 2i-MEFs. Note that Pax8 was derepressed exclusively in Dnmt3a KO 2i-MEFs. Data are presented as means \pm SD. The mean expression level of $2 \mathrm{i}-\mathrm{MEF}$ was defined as 1. Statistical analysis was performed by Student's $t$ test (WT vs 3a KO $p=0.0083$ : WT vs $3 b$ KO $p=0.0084$; two-sided, $n=3$ biologically independent samples). e DNA methylation status at promoters of representative PcG target genes that exhibited modest derepression upon Dnmt3a depletion (e.g., Hoxa2 and Pax8). Data from two independent experiments are shown. Each bar indicates a CG site, and bar height represents methylation percentage (0-100\%). Locations of genes and CGIs are indicated below.

ICF patients, we identified additional genes that were hypomethylated in both Dnmt3b KO 2i-MEFs and ICF patients (e.g., TMEM151A) (Fig. 6a,b). Taken together, our results provide candidate genes that may be involved in the pathogenesis of ICF syndrome.

We then examined the relationship between the DNMT3A mutations and DNA methylation levels at PcG target developmental genes in AML. Consistent with the assumption that DNMT3A-inactivating mutations impair repression of PcG target developmental genes, previous studies reported that some PcG target developmental genes, such as HOX genes and MEIS1, are dysregulated in AML and Dnmt3a null mouse hematopoietic stem cells ${ }^{28-30}$. To explore this further, we examined a correlation between the presence of the DNMT3A mutations and DNA methylation levels at PcG target developmental genes in AML using public datasets (GSE62298). Notably, among 30 differentially methylated PcG target developmental genes, 29 (e.g., HOXA6, HOXB3, and MEIS1) were hypomethylated in DNMT3A 
a

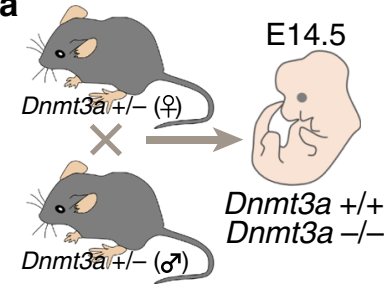

b

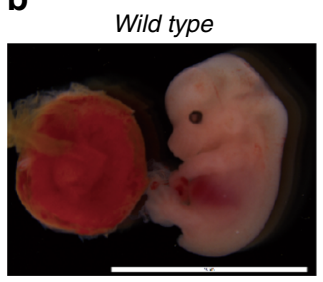

Dnmt3a KO

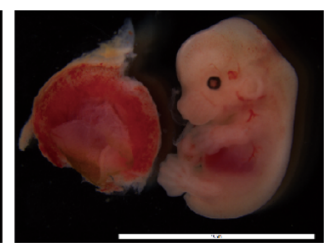

C

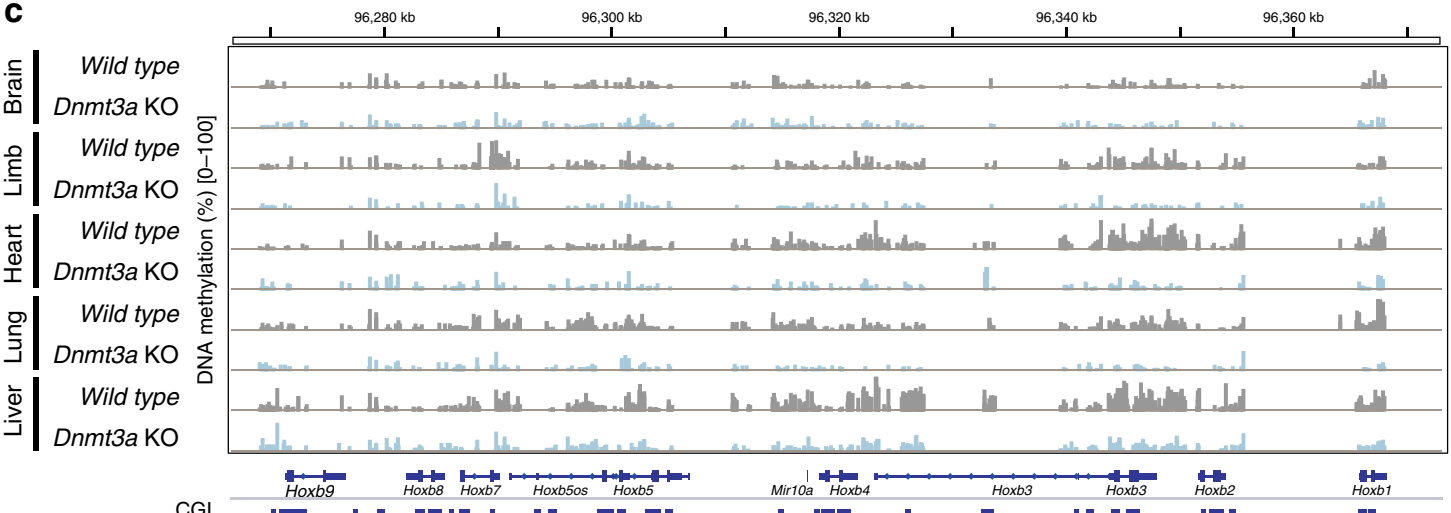
CGI

$\begin{array}{ll}\text { Brain } & \\ \text { Limb } & \text { DNA methylation } \\ \text { Methyl-seq }\end{array}$

Liver

d

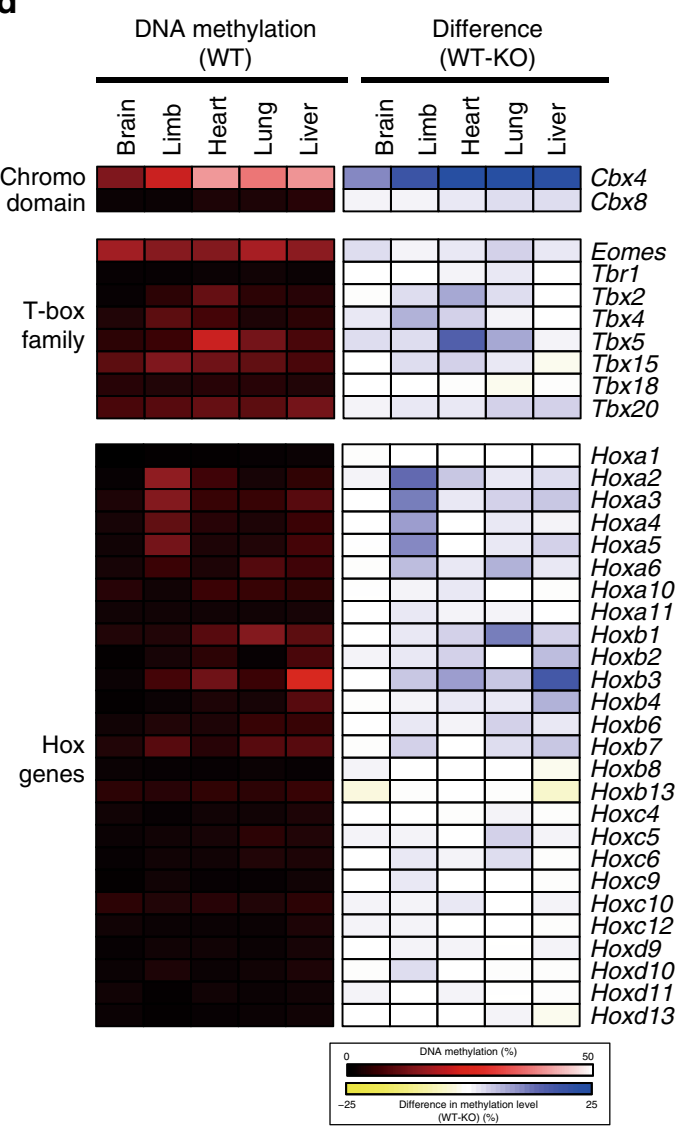

mutant AML (R882: major mutation) relative to DNMT3A WT AML, suggesting that inactivating DNMT3A mutation causes defects in epigenetic repression of PcG target developmental genes (Fig. 6c, d). These results are also consistent with those of a recent study demonstrating that a gain-of-function mutation at DNMT3A causes microcephalic dwarfism and hypermethylation of PcG-regulated genes ${ }^{31}$. Collectively, these data suggest that the e Heart
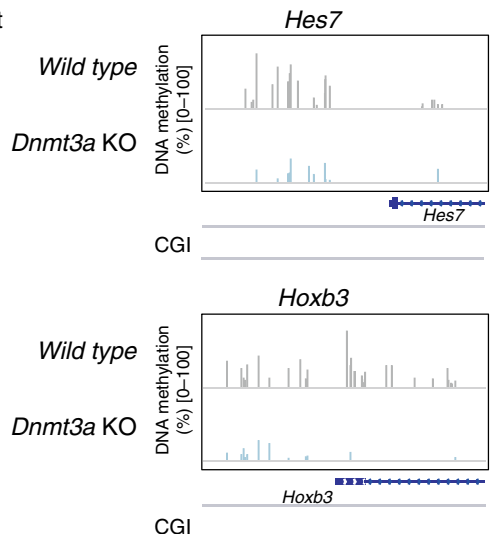

f Heart PcG target genes $(n=206)$

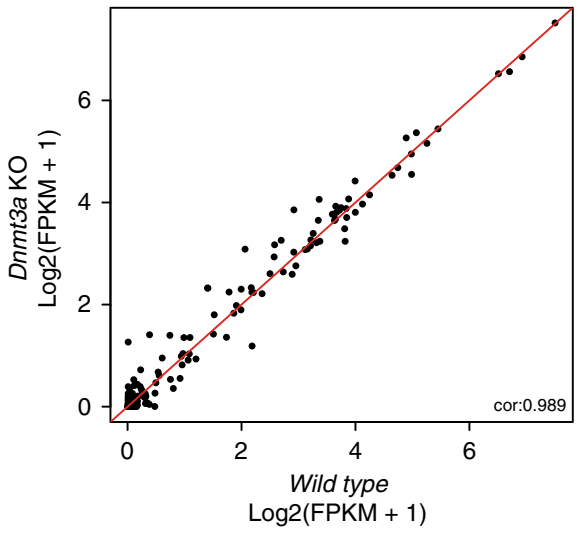

region specificity of de novo methylation by DNMT3A and DNMT3B is essentially shared between mice and humans.

\section{Discussion}

Although de novo DNA methylation plays a pivotal role during embryonic development, important unsolved questions include 
Fig. 5 Tissue-specific methylation at PcG target genes by DNMT3A. a Schematic of experimental design. Various organs (brain, limb, heart, lung, and liver) were obtained from Dnmt3a WT and KO embryos at E14.5 for subsequent analyses. b Representative image of Dnmt3a WT and KO embryos at E14.5. No obvious phenotypic changes were observed in Dnmt3a KO embryos. Scale bars, $10 \mathrm{~mm}$. c CpG methylation levels at the Hoxb cluster in Dnmt3a WT and $\mathrm{KO}$ organs (brain, limb, heart, lung, and liver), as determined by MethylC-seq. Each bar indicates a CpG site, and bar height represents methylation percentage (0-100\%). Locations of genes and CGls are indicated below. $\mathbf{d}$ The left panel shows mean DNA methylation levels at CpG sites within each representative PcG target gene in various organs, as determined by MethylC-seq. The right panel shows methylation differences between Dnmt3a WT and $\mathrm{KO}$ organs. Color scales indicate DNA methylation levels and their differences, respectively. e DNA methylation status at promoters of Hes7 and Hoxb3. Each bar indicates a CG site, and bar height represents methylation percentage, as determined by MethylC-seq (0-100\%). Locations of genes and CGIs are indicated below. $\mathbf{f}$ Expression levels (FPKM +1 ) of PcG target genes in hearts of Dnmt3a WT and KO embryos, as determined by RNA-seq.

precise roles of each DNMT3 enzyme. In this study, we utilized the hypomethylated ES cells as starting materials in a combination with genetic disruption of Dnmt3 family methyltransferases, and successfully unveiled the distinct region-specificity of de novo methylation activity of these enzymes. Consistent with a previous report, most regions are similarly methylated in WT, Dnmt3a $\mathrm{KO}$, and Dnmt $3 b \mathrm{KO}$ cells after differentiation, indicating the redundant function of DNMT3A and DNMT3B. However, we found that DNMT3B has a higher activity of de novo methylation than DNMT3A, which is remarkably pronounced at CGIs on $\mathrm{X}$ chromosome. In contrast, de novo methylation at PcG target developmental genes requires DNMT3A, which is different from the mechanism in the extraembryonic lineage. Furthermore, we showed that tissue-specific de novo methylation at PcG target developmental genes is mediated by DNMT3A and is mechanistically associated with stable repression of a small subset of the PcG target genes. Notably, no gene was identified to be hypomethylated in Dnmt3a KO epiblasts when compared with WT epiblasts. Indeed, most of identified DNMT3A target genes are unmethylated in epiblasts (Supplementary Fig. 9a, b), suggesting that de novo DNA methylation at these loci occurs after implantation. Unexpectedly, we found that DNMT3A confers gene body methylation at PcG target developmental genes, which is in contrast to other genes being methylated by DNMT3B. Moreover, we showed that human diseases harboring inactivating mutations at DNMT3 family genes exhibit reduced methylation at hypomethylated regions in mouse Dnmt3a and Dnmt3b KO cells.

Here we showed that de novo DNA methylation on the $\mathrm{X}$ chromosome mainly depends on DNMT3B but not on DNMT3A while de novo methylation at PcG target developmental genes is mediated by DNMT3A. It should be noted that the inactive $\mathrm{X}$ chromosome is heavily targeted by Polycomb complexes ${ }^{32}$, suggesting that distinct mechanisms are involved in de novo methylation at PcG target genes. Notably, more pronounced reduction in DNA methylation was observed at LINEs in Dnmt3b KO than Dnmt3a KO 2i-MEFs. Given that greater occurrence of LINEs on the $\mathrm{X}$ chromosome than on autosomes, the enriched LINEs may account for the preferential reduction in DNA methylation at the X chromosome in Dnmt3b KO 2i-MEFs.

In summary, taking advantage of DNA-hypomethylated $2 \mathrm{i} / \mathrm{L}$ ES cells, we identified a set of unique de novo target sites for DNA methylation by both DNMT3 enzymes during mammalian development. Our findings have important implications regarding the roles of de novo methyltransferases in epigenetic regulation of mammalian development and diseases.

\section{Methods}

Data reporting. No statistical methods were used to predetermine sample size. No experiments were randomized. Investigators were not blinded to allocation during the experiments.

Establishment and culture of ES cells. Zygotes with an F1 genetic background $(129 \times 1 / \mathrm{SvJ}$ and MSM/Ms) were obtained by in vitro fertilization. S/L ES and $2 \mathrm{i} / \mathrm{L}$ ES cells were established in a previous study ${ }^{16}$. Cells were maintained at $37^{\circ} \mathrm{C}$ in an atmosphere containing $5 \% \mathrm{CO}_{2}$. Briefly, S/L ES cells were established and maintained on feeders (mitomycin C-treated MEFs) in KO DMEM (GIBCO) containing $2 \mathrm{mM}$ L-glutamine, 100× NEAA, P/S, 15\% FBS, $0.11 \mathrm{mM} \beta$ mercaptoethanol (GIBCO), and $1000 \mathrm{U} / \mathrm{ml}$ human recombinant LIF (Wako). To establish S/L ES cells, a blastocyst was placed on feeders in 24-well plates (passage number 0: p0). After expansion of the ICM, the cells were passaged into 24-well plates (p1), followed by a second passage into six-well plates (p2) and a third passage into $6 \mathrm{~cm}$ dishes (p3). For derivation of 2i/L ES cells, a blastocyst was placed on feeders in 24-well plates in KO DMEM containing $2 \mathrm{mM}$ L-glutamine, $100 \times$ NEAA, P/S, 15\% FBS, $0.11 \mathrm{mM} \beta$-mercaptoethanol, $1000 \mathrm{U} / \mathrm{ml} \mathrm{LIF,} 1 \mu \mathrm{M}$ PD0325901 (STEMGENT), and $3 \mu \mathrm{M}$ CHIR99021 (STEMGENT) (p0). After passage into 24-well plates (p1), the cells were maintained without feeders in DMEM/F12 (GIBCO) and Neurobasal (GIBCO) supplemented with the B27 (GIBCO) and N2 cell supplement (GIBCO), P/S, 2 mM Glutamax (GIBCO), 1000 $\mathrm{U} / \mathrm{ml} \mathrm{LIF}, 1 \mu \mathrm{M}$ PD0325901, and $3 \mu \mathrm{M}$ CHIR99021. Cells were passaged into sixwell plates (p2) and expanded into 6-cm dishes (p3).

Genetic deletions of Dnmt3a and Dnmt3b in ES cells. Knockout of Dnmt3a or Dnmt $3 b$ for $2 \mathrm{i} / \mathrm{L}$ ES cells was performed using CRISPR/Cas9 ${ }^{16} .2 \mathrm{i} / \mathrm{L}$ ES cells at p3 were used to establish KO lines. sgRNA sequences were as follows: Dnmt $3 a$, ACCGCCTCCTGCATGATGCG; Dnmt3b: AATGCACTCCTCATACCCGC.

Establishment of 2i-MEFs by blastocyst injection. Female ICR mice were treated with pregnant mare serum gonadotropin (PMSG; $7.5 \mathrm{IU}$ ) and human chorionic gonadotropin (hCG; $7.5 \mathrm{IU}$ ) by intraperitoneal injection. Embryos were rinsed with M2 medium (Sigma) and cultured in KSOM medium until development into blastocysts. WT, Dnmt $3 a \mathrm{KO}$, and Dnmt $3 b$ KO 2i/L ES cells were trypsinized, and 7-8 cells per embryo were injected into the blastocoels of E3.5 blastocysts. Injected blastocysts were transferred into the uteri of pseudopregnant ICR mice. A Fully Automated Fluorescence Stereo Microscope Leica M205 FA (Leica MICROSYSTEMS) was used to observe GFP-labeled embryos. MEFs were isolated from E14.5 embryos and cultured in DMEM (Nacalai Tesque) with $2 \mathrm{mM}$ L-glutamine (Nacalai Tesque), 100 $\times$ nonessential amino acids (NEAA) (Nacalai Tesque), $100 \mathrm{U} /$ $\mathrm{ml}$ penicillin, $100 \mu \mathrm{g} / \mathrm{ml}$ streptomycin (P/S) (Nacalai Tesque), and $10 \%$ fetal bovine serum (FBS) (GIBCO). To remove host cells, GFP-labeled MEFs were collected by fluorescence activated cell sorting (Aria II, BD) after selection with $350 \mu \mathrm{g} / \mathrm{ml} \mathrm{G418}$ disulfate aqueous solution (Nacalai Tesque) for 7 days.

Establishment of Dnmt3a or Dnmt3I WT and KO MEFs. Dnmt3a WT and KO embryos were obtained by crossing Dnmt3a heterozygous KO (B6;129S4-Dnmt3a $<$ tm1Enl $>$ ) mice ${ }^{5}$. Dnmt3l WT and KO embryos were obtained by crossing Dnmt3l heterozygous KO mice ${ }^{12}$. MEFs were isolated from E14.5 embryos.

EpiLC induction. Culture medium for EpiLCs induction ${ }^{33}$ was DMEM/F12 + Neurobasal containing B27 and N2 cell supplements, $20 \mathrm{ng} / \mathrm{ml}$ recombinant human activin A (R\&D Systems), $12 \mathrm{ng} / \mathrm{ml}$ bFGF (Wako), $100 \mathrm{U} / \mathrm{ml}$ penicillin, $100 \mu \mathrm{g} / \mathrm{ml}$ streptomycin, and $1 \% \mathrm{KO}$ serum replacement (GIBCO).

Animals. All animal studies were conducted in compliance with ethical regulations in The University of Tokyo and Kyoto University and were approved by Institute of Medical Sciences, The University of Tokyo and Center for iPS Cell Research and Application (CiRA), Kyoto University. MSM/Ms were obtained from RIKEN Bio Resource Center ${ }^{34,35}$. B6;129S4-Dnmt3a $<$ tm1Enl $>$ mice ${ }^{5}$ were obtained from RIKEN Bio Resource Center. C57BL/6, 129×1/SvJ, ICR mice, and pseudopregnant ICR mice were purchased from SLC. Noon of the day when a plug was observed was designated as embryonic day (E) 0.5 .

Cell lines. All cell lines used for this study were derived in our laboratory and tested for mycoplasma contamination.

Sex determination. The sex of cell lines and mouse embryos was determined by PCR using primers for Sry and Uty, both of which are encoded on the Y chromosome. The primers used were as follows: Sry-Fw, CGTGGTGAGAGGCACAA 
a

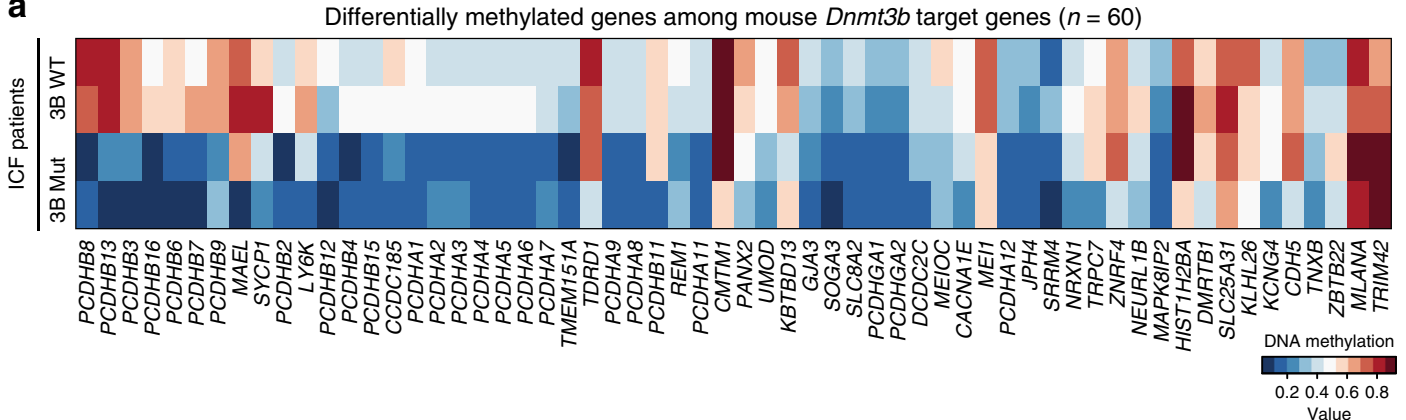

b
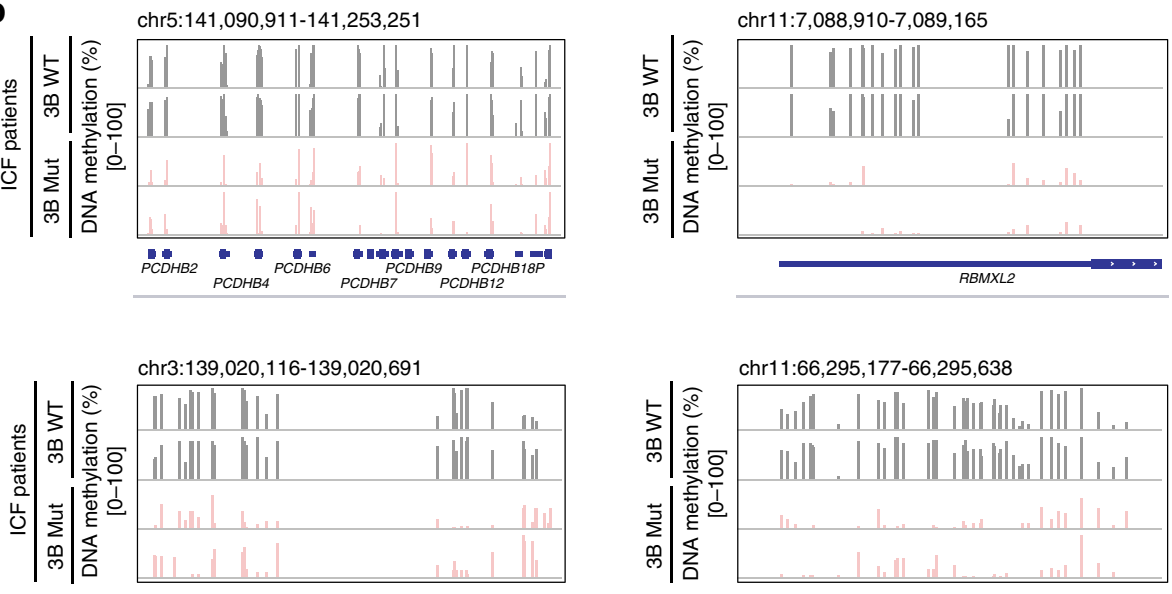

PRR23B

C

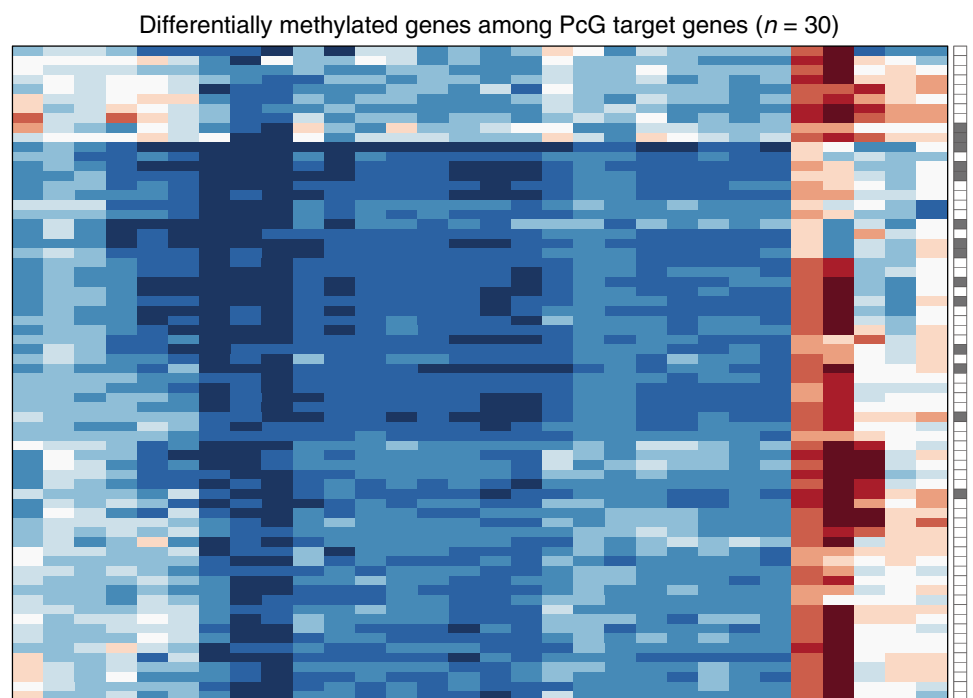

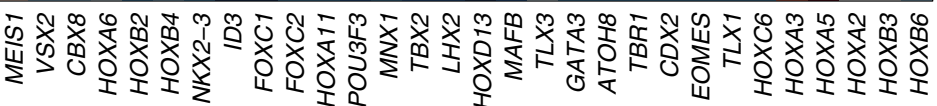

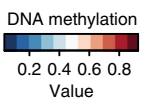

GTT; Sry-Rv, AGGCAACTGCAGGCTGTAAA; Uty-Fw, GAGTTCTTCTTGCG TTCACCATCTG; and $U t y-R v$, CTATCTAATCCACAAAGCGCCTTCTTC.

GFP labeling of ES cells. ES cells (p3) were labeled by piggyBac transposon carrying CAG-EGFP-IRES-Neo ${ }^{36}$. Plasmid and transposase $(2.5 \mu \mathrm{g}$ each) were transfected into ESCs using Xfect mESC Transfection Reagent (Clontech). Transfected cells were selected with $350 \mu \mathrm{g} / \mathrm{ml} \mathrm{G418} \mathrm{disulfate} \mathrm{aqueous} \mathrm{solution.} \mathrm{GFP} \mathrm{positive}$ colonies were picked and established as GFP-labeled clones (p5). d
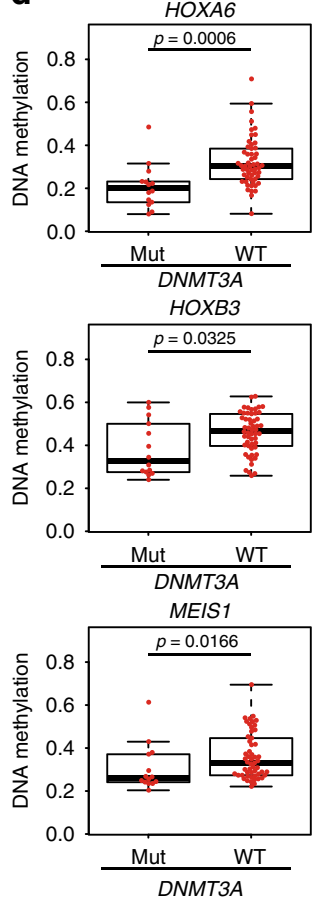

DNA/RNA extraction and CDNA synthesis. DNA was extracted using the PureLink Genomic DNA Mini Kit (Invitrogen), and RNA was extracted using the RNeasy plus Mini Kit (QIAGEN) or NucleoSpin RNA Plus (MACHEREYNAGEL). DNA and RNA were quantified on a Nanodrop 2000c (Thermo Fisher Scientific) and Qubit (Thermo Fisher Scientific). Reverse transcription was performed using the PrimeScript ${ }^{\text {ma }}$ II First strand cDNA Synthesis Kit (Takara).

Quantitative RT-PCR. Quantitative PCR was performed using GoTaq qPCR Master Mix and CXR Reference Dye (Promega) on a StepOnePlus Real-Time PCR 
Fig. 6 Reduced DNA methylation in patients with DNMT3 mutations. a Heatmap of DNA methylation levels at differentially methylated genes in mouse DNMT3B target genes in ICF syndrome patients-derived B cell lines [DNMT3B wildtype (WT): two patients, DNMT3B mutant (Mut): two patients], as determined by reduced representation bisulfite sequencing (RRBS). Genes differentially methylated ( $|\mathrm{WT}-\mathrm{Mut}|>=0.1$ ) between WT and DNMT3B Mut are shown $(n=60)$. Note that most of differentially methylated genes $(n=57)$ exhibited reduced DNA methylation in DNMT3B-mutated ICF patients. DNA methylation datasets were obtained from GSE95747 49 . b DNA methylation status at representative loci of mouse DNMT3B target genes in ICF patients. DNMT3B target genes in mice were hypomethylated similarly in DNMT3B-mutated ICF patients. Each bar indicates a CG site, and bar height represents methylation percentage, as determined by RRBS (0-100\%). Locations of genes are indicated below. c Heatmap of DNA methylation levels at PcG target developmental genes of $68 \mathrm{AML}$ patients [DNMT3A wildtype (WT): 54 patients, DNMT3A mutant (Mut): 14 patients] by Infinium assay obtained from GSE6229829. Thirty PcG target genes whose average methylation levels within gene bodies differed ( $p<0.05$; two-sided, Mann-Whitney U-test) between DNMT3A WT (white) and DNMT3A Mut (gray) are shown. Color scale indicates DNA methylation levels. $\mathbf{d}$ Box plots of DNA methylation levels within gene bodies of HOXA6, HOXB3, and MEIS1 in DNMT3A WT (54 patients) and DNMT3A Mut (14 patients) AML. Bold black lines indicate the median methylation levels. The bottom and top of the box are lower and upper quartiles, respectively. Whiskers extend to \pm 1.5 IQR. (HOXA6, $p=0.0006$ : $H O X B 3$, $p=0.0325$ : MEIS1, $p=0.0166$; two-sided, Wilcoxon signed-rank test).

system (Applied Biosystems). Transcript levels were normalized against the corresponding level of $\beta$-actin. Primers used were as follows: Pax8-Fw, ATGCCTCA CAACTCGATCAGA; Pax8-Rv, ACAATGCGTTGACGTACAACTT; $\beta$-Actin-Fw, GCCAACCGTGAAAAGATGAC; and $\beta$-Actin-Rv,

TCCGGAGTCCATCACAATG.

Ezh2 inhibitor (GSK126) treatment. Cells were seeded at a density of $2 \times 10^{5}$ cells on six-well plates the day before GSK126 treatment. The cells were then cultured in medium containing $10 \mu \mathrm{M}$ GSK126 (Funakoshi) for 2 days.

Western blot. Cells were dissolved in $500 \mu \mathrm{l}$ of RIPA lysis buffer containing protease inhibitor cocktail (Nacalai Tesque), dithiothreitol (Wako), and phosphatase inhibitor cocktail (Nacalai Tesque). For histone extraction, cells were washed with PBS and treated with trypsin for $5 \mathrm{~min}$. Cells $\left(1 \times 10^{6}\right)$ were lysed with extraction buffer on ice. The suspension was centrifuged at $12,000 \mathrm{rpm}$ for $10 \mathrm{~s}$, and the supernatant was removed. Next, $0.2 \mathrm{M} \mathrm{H}_{2} \mathrm{SO}_{4}$ was added to the pellet, and the suspension was then centrifuged at $12,000 \mathrm{rpm}$ for $20 \mathrm{~min}$. The resultant supernatant was mixed with $100 \%$ trichloroacetic acid and centrifuged at $12000 \mathrm{rpm}$ for $20 \mathrm{~min}$; the supernatant was removed. Acetone was added to the pellet and lysed. The suspension was then centrifuged at $12,000 \mathrm{rpm}$ for $20 \mathrm{~min}$, and the supernatant was removed. Finally, the pellet was lysed with $1 \times$ SDS sample buffer. Proteins were denatured with $2 \times$ SDS at $95^{\circ} \mathrm{C}$ for $5 \mathrm{~min}$. A total of $30 \mu \mathrm{g}$ of denatured protein was run on a $10 \%$ SDS/PAGE gel and transferred to Amersham Hybond-P PVDF Membrane (GE Healthcare) using PowerPac HC (Bio-Rad). Membranes were blocked in blocking buffer [ $1 \times$ TBS with $0.05 \%$ Tween-20 (TBST) containing $4 \%$ nonfat milk (Nacalai Tesque)], and then incubated overnight at $4{ }^{\circ} \mathrm{C}$ with primary antibodies diluted in blocking buffer. Primary antibodies were as follows: anti-Dnmt3a (Novus Biological, NB120-13888, 64B1446; dilution, 1:500; Santa Cruz Biotechnology, sc-20703; dilution, 1:200) and anti- $\beta$-actin (Santa Cruz Biotechnology, sc-47778; dilution, 1:1000). Blots were rinsed with TBST. Membranes were incubated for $90 \mathrm{~min}$ at room temperature with HRP-conjugated secondary antibodies. Secondary antibodies used were ECL Anti-mouse IgG, HRPlinked whole antibody from sheep (GE Healthcare, NA931V; dilution, 1:5000), and ECL Anti-rabbit IgG, HRP-linked whole antibody from donkey (GE Healthcare, NA934V; dilution, 1:5000). After rinsing with TBST, Pierce ECL plus Western Blotting Substrate (Thermo Scientific) was used for visualization, and bands were detected on a LAS4000 (GE Healthcare).

Bisulfite sequencing. DNA (200 ng) was bisulfite-treated using the EZ DNA Methylation-Gold Kit (ZYMO RESEARCH). PCR was performed with EX Taq HS (Takara). PCR products were cloned into pCR4-TOPO vector (Invitrogen). Subcloned colonies were sequenced using M13 reverse primer on an ABI 3500xL (Applied Biosystems). Primers were as follows: Hoxa1-Fw, AAGTTTGGGGTAATTTGGATAAAGG; Hoxal-Rv, CCAACCCAAAAAAAAACCTAAACTAC, Zfp467-Fw, AGGTTATTTGTTTGTGTTTAGTGTG; Zfp467-Rv, TCCAAATATA AATCTCCCAAATCTATC; Meis1-Fw, AGATAGTGAGGGTTTGAAATTTAGT AAA; and Meis1-Rv, CCACCCCTAACTCCAAATAAAAAT.

Library preparation for whole-genome bisulfite sequencing. DNA ( $500 \mathrm{ng}$, as determined by Qubit) was fragmented by sonication (Covaris) and ligated with methylated adapters supplied by TruSeq ${ }^{\mathrm{m}}$ DNA, Sample Prep Kit-v2 (Illumina). Subsequently, DNA was bisulfite-treated using EZ DNA Methylation-Gold Kit ${ }^{\text {m. }}$ Final library amplification was performed using Pfu Turbo Cx (Agilent Technologies). Sequencing libraries were assessed on a Bioanalyzer (Agilent Technologies) and quantified using the KAPA Library Quantification Kit (KAPA BIOSYSTEMS). The libraries were then sequenced on a HiSeq 2500 (Illumina) as $2 \times 100$ bp or $2 \times$ $101 \mathrm{bp}$ paired-end reads.
Library preparation for target-captured bisulfite sequencing. DNA ( $3 \mu \mathrm{g}$, as determined by Qubit) was fragmented by sonication. Subsequently, library preparation was performed using the SureSelect ${ }^{\mathrm{XT}}$ Mouse Methyl-Seq Reagent Kit (Agilent Technologies). The Methyl-Seq Kit could enrich $109 \mathrm{Mb}$ of mouse genomic regions including CpG islands, Gencode promoters, DNase I hypersensitive sites, and tissue-specific DMRs. DNA was bisulfite-treated using the EZ DNA Methylation-Gold Kit ${ }^{\mathrm{m}}$. Sequencing libraries were assessed by Bioanalyzer and quantified using the KAPA Library Quantification Kit. The libraries were then sequenced on a HiSeq 2500 (Illumina) as $2 \times 100$ bp or $2 \times 101$ bp pairedend reads.

Library preparation for RNA sequencing. RNA (200 ng) was prepared for library construction. High-quality RNA (RNA Integrity Number value $\geq 8$, as determined by Bioanalyzer) was used for library preparation. RNA-seq libraries were generated using the TruSeq Stranded mRNA LT sample prep kit (Illumina). PolyAcontaining mRNA was purified by magnetic beads conjugated to poly-T oligos, and RNA was fragmented and primed for cDNA synthesis. Cleaved RNA fragments were reverse transcribed into first-strand cDNA using transcriptase and random primers. Second-strand cDNA was synthesized by incorporation of dUTP, and ds cDNA was separated using AMPure XP beads (Beckman Coulter). A single "A" nucleotide was added to the $3^{\prime}$ ends of the blunt fragments, and adapters with index were ligated to the ends of ds cDNAs. ds cDNA fragments were amplified by PCR using PCR primer Cocktail (Illumina). The number of PCR cycles was minimized (11-15 cycles) to avoid skewing the representation of the libraries. RNA-seq libraries were sequenced on a NextSeq500 (Illumina) as 75 bp single reads.

DNA methylation data analyses. For WGBS and MethylC-seq analyses of 129/ MSM genetic background cells and tissues, SNP data for MSM/Ms were obtained from the NIG Mouse Genome Database (MSMv4HQ, http://molossinus.lab.nig.ac $\mathrm{jp} / \mathrm{msmdb} /$ index.jsp), and the MSM/Ms mouse genome was reconstructed from mm10 using these SNPs. Information about indels was not used in this study. Bases with low-quality scores and the adapters in all sequenced reads were trimmed with cutadapt-1.14 ${ }^{37}$. Trimmed reads were mapped independently to both the B6 (mm10) and MSM/Ms mouse genomes using the Bismark software-v0.18.2 ${ }^{38}$ with bowtie2 (version 2.3.0) ${ }^{39}$. Reads uniquely mapped to the same chromosome and positions of both B6 and MSM/Ms genomes were used for further analyses. B6 (129)-derived and MSM/Ms-derived sequenced reads were determined based on MSM/Ms SNP data. The Y chromosome and mitochondrial genome were omitted from the MSM/Ms reference genome due to the lack of SNP data. SNPs in CpG sites were excluded, and the patterns of bisulfite conversion were taken into account in the determination of parental alleles. Methylated cytosines were extracted from reads using the Bismark methylation extractor with the following options:-ignore 10-ignore_r2 10-ignore_3prime 5-ignore_3prime_r2 5. For MethylC-seq analysis of B6 genetic background tissues, trimmed reads were mapped to the mouse genome (mm10) using Bismark software-v0.18.2 with bowtie2 (version 2.3.0) and default settings, and uniquely mapped reads were used for extraction of methylated cytosines using the Bismark methylation extractor with options-ignore 10-ignore_3prime 5 (single-end reads) or-ignore 10-ignore_r2 10-ignore_3prime 5-ignore_3prime_r2 5. For MethylC-seq analysis, because probe sequences of the SureSelect ${ }^{\mathrm{XT}}$ were designed for the original top strand, only the original bottom data were used for this analysis. The Integrative Genomics Viewer (version 2.8.2) ${ }^{40}$ was used to visualize CpG methylated status (CpG sites at $\geq 5 \times$ coverage). Genomic locations for each genetic element were described previously ${ }^{41}$. UCSC LiftOver ${ }^{42}$ (http://genome.ucsc.edu/) was used to convert the coordinates of the $\mathrm{mm} 9$ assembly to those of the mm10 assembly.

Identification of differentially methylated loci and regions. The R/Bioconductor package DSS ${ }^{43}$ was used to identify differentially methylated loci and regions. For identification of epiblast-specific methylated loci (Fig. 1b), the DMLtest function in 
the DSS package (FDR $<0.05$, Epi $>$ ICM) was used with WGBS data (CpG sites at $\geq 5 \times$ coverage) obtained from GSE84236 to determine significantly differentially methylated loci. For identification of DNMT3A and DNMT3B target regions globally (Fig. $2 \mathrm{a}$ and Supplementary Data 2), the DMLtest function in the DSS package $(\mathrm{FDR}<0.01$ and difference in methylation levels $(\mathrm{WT}-\mathrm{KO})>0.4$ ) was used with $1 \mathrm{k}$ tiled $\mathrm{CpG}$ methylation WGBS data (CpG sites at $\geq 10 \times$ coverage) to determine significantly differentially methylated tiles (DMTs; Supplementary Data 1). For identification of DNMT3A and DNMT3B target genes (Fig. 2b, c, Supplementary Fig. 3a, b and Supplementary Data 1-5), the DMLtest and callDMR functions in the DSS package (delta $=0.2$, p.threshold $=0.00001$, $\operatorname{minCG}=10$ ) were used with MethylC-seq data (CpG sites at $\geq 50 \times$ coverage) to determine significantly differentially methylated regions (DMRs, each KO vs WT, Supplementary Data 2 and 4). The genes included in the DMRs (WT $>$ KO) were defined as target genes (Supplementary Data 3 and 5). Gene locations were obtained from the GENCODE annotation file (vM15).

RNA-seq data analyses. After trimming of adaptor sequences and low-quality bases with cutadapt $-1.15^{37}$, sequenced reads were mapped to the mouse reference genome (mm10) using tophat-2.1.1 ${ }^{44}$ with the GENCODE version M15 annotation.gtf file and the aligner Bowtie2-2.3.239. Uniquely mapped reads (MAPQ $\geq 20$ ) were used for further analyses. The expression level of each gene was calculated as fragments per kilobase per million mapped reads (FPKM) using cufflinks-2.2.1 ${ }^{45}$.

ChIP-seq data analysis. Raw ChIP-seq data (Dnmt3a and Dnmt3b binding data) in ES cells were obtained from GSE57413 ${ }^{14}$. Adaptors and low-quality bases at the $3^{\prime}$ ends of reads were trimmed using cutadapt- $1.15^{37}$. Untrimmed and trimmed reads of $20 \mathrm{bp}$ or more were mapped to the mouse genome (mm10) with BWA $0.7 .12^{46}$. Duplicate reads, chimeric reads, and reads with low mapping quality $(\mathrm{MAPQ}<20)$ were removed using Picard (http://broadinstitute.github.io/picard/) and SAMtools ${ }^{47}$. Reads mapped to blacklisted regions (https://sites.google.com/ site/anshulkundaje/projects/blacklists) were excluded from further analysis. Average ChIP-seq plots were drawn using ngsplot-2.47 ${ }^{48}$.

Reporting summary. Further information on research design is available in the Nature Research Life Sciences Reporting Summary linked to this article.

\section{Data availability}

All data analyzed by WGBS, MethylC-seq, and RNA-seq were deposited in the Gene Expression Omnibus (GEO) under accession number GSE111172. MethylC-seq data from Dnmt3a WT tissue were deposited under accession number GSE111173. The publicly available datasets used in this study are: GSE84236, GSE95747, GSE62298, GSE57413, GSE96529, GSE95747, GSE62298. All other relevant data supporting the key findings of this study are available within the article and its Supplementary Information files or from the corresponding authors upon reasonable request. The source data underlying Supplementary Fig $4 \mathrm{c}$ are provided as a Source Data file. A reporting summary for this Article is available as a Supplementary Information file.

Received: 1 August 2019; Accepted: 2 June 2020;

Published online: 24 June 2020

\section{References}

1. Smith, Z. D. \& Meissner, A. DNA methylation: roles in mammalian development. Nat. Rev. Genet. 14, 204-220 (2013).

2. Bird, A. DNA methylation patterns and epigenetic memory. Genes Dev. 16, 6-21 (2002).

3. Jaenisch, R. \& Bird, A. Epigenetic regulation of gene expression: how the genome integrates intrinsic and environmental signals. Nat. Genet. 33(Suppl), 245-254 (2003).

4. Jackson, M. et al. Severe global DNA hypomethylation blocks differentiation and induces histone hyperacetylation in embryonic stem cells. Mol. Cell Biol. 24, 8862-8871 (2004).

5. Okano, M., Bell, D. W., Haber, D. A. \& Li, E. DNA methyltransferases Dnmt3a and Dnmt3b are essential for de novo methylation and mammalian development. Cell 99, 247-257 (1999).

6. Li, E., Bestor, T. H. \& Jaenisch, R. Targeted mutation of the DNA methyltransferase gene results in embryonic lethality. Cell 69, 915-926 (1992).

7. Jin, B. et al. DNA methyltransferase 3B (DNMT3B) mutations in ICF syndrome lead to altered epigenetic modifications and aberrant expression of genes regulating development, neurogenesis and immune function. Hum. Mol. Genet. 17, 690-709 (2008).

8. Shah, M. Y. \& Licht, J. D. DNMT3A mutations in acute myeloid leukemia. Nat. Genet. 43, 289-290 (2011).
9. Smith, Z. D. et al. A unique regulatory phase of DNA methylation in the early mammalian embryo. Nature 484, 339-344 (2012).

10. Smith, Z. D. et al. Epigenetic restriction of extraembryonic lineages mirrors the somatic transition to cancer. Nature 549, 543-547 (2017).

11. Bourc'his, D., Xu, G. L., Lin, C. S., Bollman, B. \& Bestor, T. H. Dnmt3L and the establishment of maternal genomic imprints. Science 294, 2536-2539 (2001).

12. Hata, K., Okano, M., Lei, H. \& Li, E. Dnmt3L cooperates with the Dnmt3 family of de novo DNA methyltransferases to establish maternal imprints in mice. Development 129, 1983-1993 (2002).

13. Kaneda, M. et al. Essential role for de novo DNA methyltransferase Dnmt3a in paternal and maternal imprinting. Nature 429, 900-903 (2004).

14. Baubec, T. et al. Genomic profiling of DNA methyltransferases reveals a role for DNMT3B in genic methylation. Nature 520, 243-247 (2015).

15. Manzo, M. et al. Isoform-specific localization of DNMT3A regulates DNA methylation fidelity at bivalent CpG islands. EMBO J. 36, 3421-3434 (2017).

16. Yagi, M. et al. Derivation of ground-state female ES cells maintaining gametederived DNA methylation. Nature 548, 224-227 (2017).

17. Hackett, J. A. \& Surani, M. A. Regulatory principles of pluripotency: from the ground state up. Cell Stem Cell 15, 416-430 (2014).

18. Shirane, K. et al. Global landscape and regulatory principles of DNA methylation reprogramming for germ cell specification by mouse pluripotent stem cells. Dev. Cell 39, 87-103 (2016).

19. Auclair, G., Guibert, S., Bender, A. \& Weber, M. Ontogeny of CpG island methylation and specificity of DNMT3 methyltransferases during embryonic development in the mouse. Genome Biol. 15, 10.1186/s13059-014-0545-5 (2014).

20. Gendrel, A. V. et al. Smchd1-dependent and -independent pathways determine developmental dynamics of $\mathrm{CpG}$ island methylation on the inactive X chromosome. Dev. Cell 23, 265-279 (2012).

21. Liao, J. et al. Targeted disruption of DNMT1, DNMT3A and DNMT3B in human embryonic stem cells. Nat. Genet. 47, 469-478 (2015).

22. Toyoda, S. et al. Developmental epigenetic modification regulates stochastic expression of clustered protocadherin genes, generating single neuron diversity. Neuron 82, 94-108 (2014).

23. Boyer, L. A. et al. Polycomb complexes repress developmental regulators in murine embryonic stem cells. Nature 441, 349-353 (2006).

24. Jones, P. A. Functions of DNA methylation: islands, start sites, gene bodies and beyond. Nat. Rev. Genet. 13, 484-492 (2012).

25. Neri, F. et al. Intragenic DNA methylation prevents spurious transcription initiation. Nature 543, 72-77 (2017)

26. Csankovszki, G., Nagy, A. \& Jaenisch, R. Synergism of Xist RNA, DNA methylation, and histone hypoacetylation in maintaining $\mathrm{X}$ chromosome inactivation. J. Cell Biol. 153, 773-784 (2001).

27. Brunetti, L., Gundry, M. C. \& Goodell, M. A. DNMT3A in Leukemia. Cold Spring Harb Perspect Med 7, 10.1101/cshperspect.a030320 (2017).

28. Challen, G. A. et al. Dnmt3a is essential for hematopoietic stem cell differentiation. Nat. Genet. 44, 23-31 (2011).

29. Ferreira, H. J. et al. DNMT3A mutations mediate the epigenetic reactivation of the leukemogenic factor MEIS1 in acute myeloid leukemia. Oncogene 35, 3079-3082 (2016)

30. Tan, Y. T. et al. Deregulation of HOX genes by DNMT3A and MLL mutations converges on BMI1. Leukemia 30, 1609-1612 (2016).

31. Heyn, P. et al. Gain-of-function DNMT3A mutations cause microcephalic dwarfism and hypermethylation of Polycomb-regulated regions. Nat Genet. 10.1038/s41588-018-0274-x (2018).

32. Plath, K. et al. Role of histone $\mathrm{H} 3$ lysine 27 methylation in $\mathrm{X}$ inactivation. Science 300, 131-135 (2003).

33. Hayashi, K., Ohta, H., Kurimoto, K., Aramaki, S. \& Saitou, M. Reconstitution of the mouse germ cell specification pathway in culture by pluripotent stem cells. Cell 146, 519-532 (2011).

34. Takada, T. et al. The ancestor of extant Japanese fancy mice contributed to the mosaic genomes of classical inbred strains. Genome Res 23, 1329-1338 (2013).

35. Takada, T., Yoshiki, A., Obata, Y., Yamazaki, Y. \& Shiroishi, T. NIG_MoG: a mouse genome navigator for exploring intersubspecific genetic polymorphisms. Mamm. Genome 26, 331-337 (2015).

36. Kim, S. I. et al. Inducible transgene expression in human iPS cells using versatile all-in-one piggyBac transposons. Methods Mol. Biol. 1357, 111-131 (2016).

37. Martin, M. Cutadapt removes adapter sequences from high-throughput sequencing reads. 2011 17, 10-12, 10.14806/ej.17.1.200 (2011).

38. Krueger, F. \& Andrews, S. R. Bismark: a flexible aligner and methylation caller for Bisulfite-Seq applications. Bioinformatics 27, 1571-1572 (2011).

39. Langmead, B. \& Salzberg, S. L. Fast gapped-read alignment with Bowtie 2. Nat. Methods 9, 357-359 (2012).

40. Robinson, J. T. et al. Integrative genomics viewer. Nat. Biotechnol. 29, 24-26 (2011). 
41. Illingworth, R. S. et al. Orphan CpG islands identify numerous conserved promoters in the mammalian genome. Plos Genet. 6, e1001134 (2010).

42. Rosenbloom, K. R. et al. The UCSC Genome Browser database: 2015 update. Nucleic Acids Res 43, D670-D681 (2015).

43. $\mathrm{Wu}, \mathrm{H}$. et al. Detection of differentially methylated regions from wholegenome bisulfite sequencing data without replicates. Nucleic Acids Res $\mathbf{4 3}$ e141 (2015).

44. Kim, D. et al. TopHat2: accurate alignment of transcriptomes in the presence of insertions, deletions and gene fusions. Genome Biol. 14, R36 (2013).

45. Trapnell, C. et al. Transcript assembly and quantification by RNA-Seq reveals unannotated transcripts and isoform switching during cell differentiation. Nat. Biotechnol. 28, 511-515 (2010).

46. Li, H. \& Durbin, R. Fast and accurate short read alignment with BurrowsWheeler transform. Bioinformatics 25, 1754-1760 (2009).

47. Li, H. et al. The sequence alignment/Map format and SAMtools. Bioinformatics 25, 2078-2079 (2009).

48. Shen, L., Shao, N., Liu, X. \& Nestler, E. ngs.plot: quick mining and visualization of next-generation sequencing data by integrating genomic databases. BMC Genomics 15, 284 (2014).

49. Gatto, S. et al. ICF-specific DNMT3B dysfunction interferes with intragenic regulation of mRNA transcription and alternative splicing. Nucleic Acids Res. 45, 5739-5756 (2017).

\section{Acknowledgements}

We are grateful to S. Sakurai and D. Seki for technical assistance. Y. Y. was supported in part by P-CREATE (JP18cm0106203h0003), Japan Agency for Medical Research and Development (AMED); AMED-CREST (JP19gm1110004h9903), AMED; JSPS

KAKENHI (18H04026, 20H05384); the Princess Takamatsu Cancer Research Fund; the Takeda Science Foundation; the Mochida Foundation; and the Naito Foundation. T.Y. was supported by AMED-CREST (19gm1110004s0203); JSPS KAKENHI 19H03418; Core Center for iPS Cell Research, Research Center Network for Realization of Regenerative Medicine, AMED; and the iPS Cell Research Fund. M. Y. was supported by JSPS KAKENHI 15J05792. The ASHBi is supported by World Premier International Research Center Initiative (WPI), MEXT, Japan.

\section{Author contributions}

M.Y., T.Y., and Y.Y. designed and conceived the study and wrote the manuscript. M.Y. and T.U. generated cell lines, performed experiments, and generated WGBS and
Methyl-seq libraries. A.T. performed blastocyst injections. K.N. and K.H. provided Dnmt3l WT and KO MEFs. M.K., S.O., M.S., A.M., and T.Y. analyzed ChIP-seq, WGBS, Methyl-seq, and RNA-seq data in mouse and human.

\section{Competing interests}

The authors declare no competing interests.

\section{Additional information}

Supplementary information is available for this paper at https://doi.org/10.1038/s41467020-16989-w.

Correspondence and requests for materials should be addressed to T.Y. or Y.Y.

Peer review information Nature Communications thanks Hongshan Guo and the other, anonymous, reviewer(s) for their contribution to the peer review of this work. Peer reviewer reports are available

Reprints and permission information is available at http://www.nature.com/reprints

Publisher's note Springer Nature remains neutral with regard to jurisdictional claims in published maps and institutional affiliations.

(c) (i) Open Access This article is licensed under a Creative Commons Attribution 4.0 International License, which permits use, sharing, adaptation, distribution and reproduction in any medium or format, as long as you give appropriate credit to the original author(s) and the source, provide a link to the Creative Commons license, and indicate if changes were made. The images or other third party material in this article are included in the article's Creative Commons license, unless indicated otherwise in a credit line to the material. If material is not included in the article's Creative Commons license and your intended use is not permitted by statutory regulation or exceeds the permitted use, you will need to obtain permission directly from the copyright holder. To view a copy of this license, visit http://creativecommons.org/ licenses/by/4.0\%

(C) The Author(s) 2020 